\title{
Netrin-1 promotes naive pluripotency through Neo1 and Unc5b co-regulation of Wnt and MAPK signalling
}

\author{
Aurélia Huyghe ${ }^{1,13}$, Giacomo Furlan ${ }^{1,13}$, Duygu Ozmadenci, ${ }^{1,13}$, Christina Galonska2, Jocelyn Charlton 2,3,4,5, \\ Xavier Gaume', Noémie Combémorel ${ }^{1}$, Christina Riemenschneider ${ }^{2}$, Nicolas Allègre ${ }^{6}$, Jenny Zhang ${ }^{7}$, \\ Pauline Wajda', Nicolas Rama ${ }^{8}$, Pauline Vieuguée ${ }^{8}$, Isabelle Durand ${ }^{9}$, Marie Brevet ${ }^{10}{ }^{10}$, Nicolas Gadot ${ }^{10}$, \\ Thomas Imhof ${ }^{11}$, Bradley J. Merrill ${ }^{7}{ }^{7}$, Manuel Koch ${ }^{11}{ }^{11}$, Patrick Mehlen ${ }^{8,12}$, Claire Chazaud ${ }^{6}$, \\ Alexander Meissner (iD) 2,3,4,5 and Fabrice Lavial $1{ }^{1 凶}$
}

In mouse embryonic stem cells ( $\mathrm{mESCs}$ ), chemical blockade of Gsk3 $\alpha / \beta$ and Mek1/2 (2i) instructs a self-renewing ground state whose endogenous inducers are unknown. Here we show that the axon guidance cue Netrin-1 promotes naive pluripotency by triggering profound signalling, transcriptomic and epigenetic changes in mESCs. Furthermore, we demonstrate that Netrin-1 can substitute for blockade of Gsk3 $\alpha / \beta$ and Mek1/2 to sustain self-renewal of $\mathrm{mESCs}$ in combination with leukaemia inhibitory factor and regulates the formation of the mouse pluripotent blastocyst. Mechanistically, we reveal how Netrin-1 and the balance of its receptors Neo1 and Unc5B co-regulate Wnt and MAPK pathways in both mouse and human ESCs. Netrin-1 induces Fak kinase to inactivate Gsk3 $\alpha / \beta$ and stabilize $\beta$-catenin while increasing the phosphatase activity of a Ppp2r2c-containing Pp2a complex to reduce Erk1/2 activity. Collectively, this work identifies Netrin-1 as a regulator of pluripotency and reveals that it mediates different effects in mESCs depending on its receptor dosage, opening perspectives for balancing self-renewal and lineage commitment.

M ouse embryonic stem cells (mESC) are mainly regulated by four signalling cues ${ }^{1-3}$. Leukaemia inhibitory factor $(\text { lif })^{4}$, Wnt $3 \mathrm{a}^{5}$ and $\mathrm{Bmp} 4^{6}$ sustain self-renewal, whereas fibroblast growth factor 4 (Fgf4) triggers exit from self-renewal via Erk1/2 activation ${ }^{7}$. Conventional culture conditions require Lif and serum or knockout serum replacement (hereafter serum/Lif) to maintain a self-renewing state. In this state, the Fgf-MAPK and the repressive Gsk3 $\alpha / \beta$-Tcf7ll pathways remain active, leading to a heterogeneous population of cells. These metastable mESCs exhibit fluctuating expression of Nanog and detectable levels of lineage-affiliated genes ${ }^{8}$. The suppression of MAPK signalling (via Mek1/2 blockade) and activation of the Wnt pathway (via Gsk $3 \alpha / \beta$ blockade) (hereafter $2 \mathrm{i}$ ), supports self-renewal of mESCs and instructs a ground state of pluripotency ${ }^{8-10}$. The $2 \mathrm{i}$ mESCs display uniform Nanog expression and negligible levels of lineage-affiliated genes. This finding has led to the establishment of germline-competent ESCs from recalcitrant mouse strains and from rat $^{11,12}$. However, the prolonged blockade of Mek1/2 has been shown to compromise mESC genomic stability, calling into question the use of $2 \mathrm{i}^{13}$. Thus, the identification of endogenous pathways controlling naive pluripotency is crucial not only for advancing our understanding of embryonic development but also for best developing strategies to generate stable human naive pluripotent cells.

Netrins are secreted proteins that have been identified as having a role in axon guidance during nervous system development $^{14,15}$. Netrin-1 (encoded by the Ntn1 gene), which was initially purified as a soluble laminin-related molecule that elicited the growth of commissural axons, is now considered as a pleiotropic ligand involved in development and diseases ${ }^{14,16,17}$. Most functions of Netrin-1 are mediated through the receptors deleted in colorectal carcinoma (Dcc), Unc5 homologues (that is, Unc5A, Unc5B, Unc5C and Unc5D) and neogenin (Neo1 ${ }^{18-20}$. The characterization of this repertoire led to identification of Netrin-1 as a bifunctional molecule that exerts opposing effects-attracting or repelling-neurons, endothelial or immune cells, depending on the receptors it engages ${ }^{21-23}$. Our previous work showed that it constrains apoptosis during reprogramming ${ }^{24}$ but the function of this pathway in self-renewal and lineage commitment remains unknown.

'Cellular Reprogramming and Oncogenesis Laboratory, Equipe labellisée la Ligue contre le cancer, Labex DEVweCAN, Université de Lyon, Université Claude Bernard Lyon 1, INSERM 1052, CNRS 5286, Centre Léon Bérard, Centre de recherche en cancérologie de Lyon, Lyon, France. ${ }^{2}$ Department of Genome Regulation, Max Planck Institute for Molecular Genetics, Berlin, Germany. ${ }^{3}$ Broad Institute of MIT and Harvard, Cambridge, MA, USA. ${ }^{4 H a r v a r d ~ S t e m ~}$ Cell Institute, Cambridge, MA, USA. ${ }^{5}$ Department of Stem Cell and Regenerative Biology, Harvard University, Cambridge, MA, USA. ${ }^{6}$ GReD, Université Clermont Auvergne, CNRS, INSERM, BP38, Clermont-Ferrand, France. ${ }^{7}$ Department of Biochemistry and Molecular Genetics, University of Illinois at Chicago, Chicago, IL, USA. ${ }^{8}$ Apoptosis, Cancer and Development Laboratory, Université de Lyon, Université Claude Bernard Lyon 1, INSERM 1052 , CNRS 5286, Centre Léon Bérard, Centre de recherche en cancérologie de Lyon, Lyon, France. ${ }^{9}$ Cytometry Facility, Université de Lyon, Université Claude Bernard Lyon 1, Centre Léon Bérard, Centre de recherche en cancérologie de Lyon, INSERM 1052, CNRS 5286, Lyon, France. ${ }^{10}$ Research Pathology platform, Department of translational research and innovation, Centre Léon Bérard, Lyon, France. "Institute for Dental Research and Oral Musculoskeletal Research, Center for Biochemistry, University of Cologne, Cologne, Germany. ${ }^{12}$ Department of Translational Research and Innovation, Centre Léon Bérard, Lyon, France. ${ }^{13}$ These authors contributed equally: Aurélia Huyghe, Giacomo Furlan, Duygu Ozmadenci. ${ }^{凶}$ e-mail: fabrice.lavial@lyon.unicancer.fr 
Here, we reveal an early developmental function for Netrin-1 in the control of naive pluripotency. We found that Netrin-1 and its receptors Neol and Unc5B control Wnt and MAPK signalling in mESCs and support self-renewal in combination with Lif. In vivo, Netrin-1 regulates the formation of the pluripotent compartment of mouse preimplantation embryos. Our findings shed light on an unexpected regulator of pluripotency and reveal that a single ligand can have diverse effects in stem cells depending on its repertoire of receptors.

\section{Results}

Netrin-1 is regulated by Wnt and MAPK signalling in mouse and human pluripotent stem cells. To identify regulators of naive pluripotency, we compared the transcriptomes of serum/Lif mESCs supplemented with inhibitors of Gsk3 $\alpha / \beta$ (CHIR99021), Mek1/2 (PD0325901) or both (2i) for $48 \mathrm{~h}$. Among the transcripts affected by modulation of Wnt and MAPK signalling, Ntn1 was induced by Gsk $3 \alpha / \beta$ inhibition and by $2 \mathrm{i}$ but repressed by Mek $1 / 2$ blockade at both transcript and protein levels (Fig. 1a,b), whereas other Netrin family members (Netrin-4, Netrin-5, Netrin-G1 and Netrin-G2) ${ }^{25}$ remained unaffected (Extended Data Fig. 1a). Since Netrin-1 is expressed at basal levels in serum/Lif but is elevated following Gsk3 $\alpha / \beta$ inhibition (Fig. 1a,b), we investigated whether it constitutes a target of Wnt. Consistently, mESCs treated with recombinant Wnt3a increased Netrin-1 levels, whereas treatment with Lif and Bmp4 had no effect (Fig. 1c). Netrin-1 induction was also observed in human induced pluripotent stem (hiPS) cells in response to Gsk3 $\alpha / \beta$ inhibition or Wnt3a stimulation (Extended Data $1 b)$. Since canonical Wnt stimulation has been shown to alleviate the repressive effect of Tcf7l1 ${ }^{26}$, we evaluated Netrin-1 expression in mESCs lacking Tcf7L1 and/or Lef1 (J.Z., B.R. Shy and B.J.M., manuscript in revision). Netrin-1 was released in the absence of Tcf7l1 but reduced if both Tcf7l1 and Lef1 were depleted, indicating that Tcf7l1 acts as a repressor of Netrin-1 activation by Lef1 (Extended Data Fig. 1c,d). Collectively, these results demonstrate that Netrin-1 is regulated by Wnt and MAPK signalling in pluripotent stem cells.

The activation of the Netrin-1-Neo1-Unc5B signalling axis sustains Nanog and mESC undifferentiated state. The distribution of naive pluripotency factors such as Nanog is heterogeneous in serum/Lif and becomes homogeneous with $2 \mathrm{i}^{9}$. Using $N t n 1^{\text {pgeo }}$ knock-in reporter mESCs ${ }^{27}$, we found that Netrin-1 expression is confined to $8 \%$ of serum/Lif mESCs, confirming its basal expression in this condition. This $\beta$-galactosidase-positive fraction increased to 26 and $23 \%$ in the presence of Gsk $3 \alpha / \beta$ inhibitor and $2 \mathrm{i}$, respectively (Fig. 1d). Exploration of single-cell transcriptomic data ${ }^{28}$ showed similar results (Extended Data Fig. 1e) but revealed that the mean Ntn1 expression level per cell is higher with 2i (Extended Data Fig. 1f).

Due to its induction with $2 \mathrm{i}$, we investigated whether Netrin-1 could actively instruct ground-state pluripotency features. In serum/Lif mESCs, mouse induced pluripotent stem cells (miPSCs) and preimplantation embryos, the Dcc receptor was not expressed but Unc5B and Neol were detected (Extended Data Fig. 2a,b). We therefore generated mESCs that exogenously express different haemagglutinin (HA)-tagged Netrin-1 forms from Cre-excisable transgenes (Fig. 1e), wild-type (WT) Netrin-1 or Netrin-1 mutated on residues critical for its interaction with the Dcc-related protein Neo1 (Netrin-1(Neo1-mut)) or with Unc5B (Netrin-1(Unc5Bmut) $)^{17,20}$ (Fig. 1f,g). We established mESC monoclones expressing the Netrin-1 proteins at similar levels (Extended Data Fig. 2c), comparable to its level in 2i (Extended Data Fig. 2d). Fluorescenceactivated cell sorting (FACS) analysis showed that the size and granulometry of Netrin-1(WT) cells became homogeneous, with a prominent contribution of Unc5B (Extended Data Fig. 2e). We next revealed that Nanog heterogeneity was reduced in Netrin-1(WT) mESCs grown in serum/Lif (Fig. 1h,i). This observation was associated with increased levels of Esrrb and Sox2, whereas Oct4 levels remained constant (Fig. 1j). Both Netrin-1 mutants failed to confer this effect, highlighting the complementary roles of Neol and Unc5B (Fig. 1h-j). Transcriptomic data of single mESCs grown in serum/Lif also revealed a correlation between Ntn1 and Esrrb levels ${ }^{29}$ (Extended Data Fig. 2f).

We next assessed whether activation of Netrin-1 signalling, by sustaining Nanog, safeguards the undifferentiated state. When cells were grown without Lif for $7 \mathrm{~d}$ and replated for $7 \mathrm{~d}$ with Lif (Fig. 1k), expression of Netrin-1(WT) conferred strong resistance to differentiation, whereas both mutants failed to do so, reinforcing the involvement of both receptors in Netrin-1 function (Fig. 11,m). As expected, excision of the Netrin-1 transgene abrogated the effect in revertant cells (Fig. 1n,o). mESCs with doxycyxline (dox)-inducible (doxi) Netrin-1 expression also presented enhanced resistance to differentiation upon dox addition (Extended Data Fig. 2g-i).

We next demonstrated that sustained expression of Netrin-1 severely impairs mESC differentiation. We first evaluated whether Netrin-1 maintains the expression of naive pluripotency markers in differentiation conditions. Nanog and Esrrb were still expressed in Netrin-1(WT) mESCs after $6 \mathrm{~d}$ of culture in N2B27 medium without Lif, but were not expressed in control cells (Fig. 1p). Immunofluorescence confirmed that some Netrin-1(WT) mESCs

Fig. 1 | Netrin-1 signalling controls pluripotency features. a, Ntn1 transcript levels in mESCs grown as indicated. Data are $\log _{2}$ FPKM values normalized to serum/Lif mESCs. Inh, inhibitor. b. Western blots performed in similar settings as a. c, Western blot in mESCs cultured in serum/Lif as indicated (3 independent experiments). d, Representative bright-field images of $N t n 7^{7 \mathrm{geo}} \mathrm{mESC}$ s grown as in a. Scale bars, $250 \mu \mathrm{m}$. Percentages of positive cells are indicated. $n$ is the total number of cells counted. $\mathbf{e}$, Schematic of the approach. The mESCs were stably transfected with the depicted constructs. IRES, internal ribosome entry site; Pac, puromycin N-acetyltransferase; pA, polyA. f, Netrin-1 protein structure. LN, laminin-like domain; LE, EGF repeats. g, Representation of the Netrin-1 mESC mutants. Netrin-1(Neo1-mut) harbours the L111E mutation, whereas Netrin-1(Unc5B-mut) contains R348A/ R349A/R351A mutations. h, Nanog and Oct4 immunofluorescence on indicated mESCs. Scale bars, $50 \mu \mathrm{m}$. i, Quantification of the Nanog/Oct4 intensity ratio. $n$ is the number of cells analysed. The centre line represents the median of the data, the box edges represent upper and lower quartiles, whiskers show highest and lowest values excluding outliers. Two-tailed Student's $t$-test. $\mathbf{j}$, Western blot on indicated mESCs. $\mathbf{k}$, Scheme depicting assays for exit from pluripotency. I, $\mathbf{m}$, Colony counts for alkaline phosphatase activity (I) and images from a representative experiment ( $\mathbf{m}$ ) from assays for exit from pluripotency. Data are mean \pm s.d., $n=3$ independent experiments; two-tailed Student's $t$-test. $\mathbf{n}, \mathbf{o}$, Assays for exit from pluripotency. $\mathbf{n}$, Images from a representative experiment ( $n=3$ independent experiments). o, Colony counts. Data are mean \pm s.d., $n=3$ independent experiments; two-tailed Student's $t$-test. p. Western blot for Nanog and Esrrb after $6 \mathrm{~d}$ in N2B27 (-Lif) ( $n=3$ independent experiments). q, r, Nanog and Esrrb immunofluorescence after $6 \mathrm{~d}$ in N2B27 (-Lif). q, Representative images. Bars: $50 \mu \mathrm{m}$. $\mathbf{r}$, Counts of Nanog- and Esrrb-positive cells. Data are mean \pm s.d., $n=3$ independent experiments; two-tailed Student's t-test; 488 control and 416 Netrin-1(WT) mESCs. s, Western blot of embryoid bodies ( $n=3$ independent experiments). $\mathbf{t}$, Histological analysis of teratoma. Scale bars, $250 \mu \mathrm{m}$. Four independent teratomas were analysed per cell line. u,v, Long-term self-renewal assays. u, Colony counts, normalized to the number of colonies formed by control cells for each passage (P) (red dotted line). $\mathbf{v}$, Images from a representative experiment ( $n=2$ independent experiments). 
sustain Nanog and Esrrb expression in differentiation-promoting conditions (Fig. 1q,r). Similarly, after $7 \mathrm{~d}$ in non-adherent culture conditions, embryoid bodies derived from Netrin-1(WT) mESCs failed to repress Nanog or Esrrb expression (Fig. 1s) or to induce differentiation genes (Extended Data Fig. 2j). Teratoma assays also revealed a severe differentiation defect caused by sustained expression of Netrin-1 (Fig. 1t). Finally, when cells were grown at clonal density on laminin in N2B27 medium with Lif for five passages, Netrin-1 expression increased the self-renewal ability of mESCs $($ Fig. $1 \mathrm{u}, \mathrm{v})$. Collectively, these results showed that the

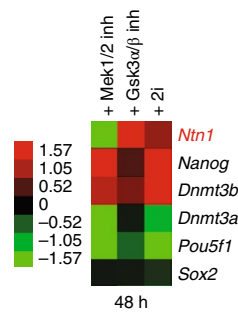

e

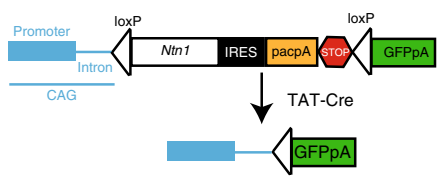

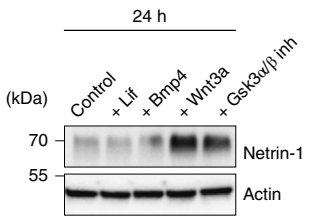

$\mathbf{f}$

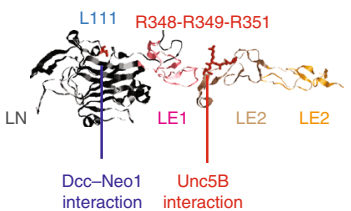

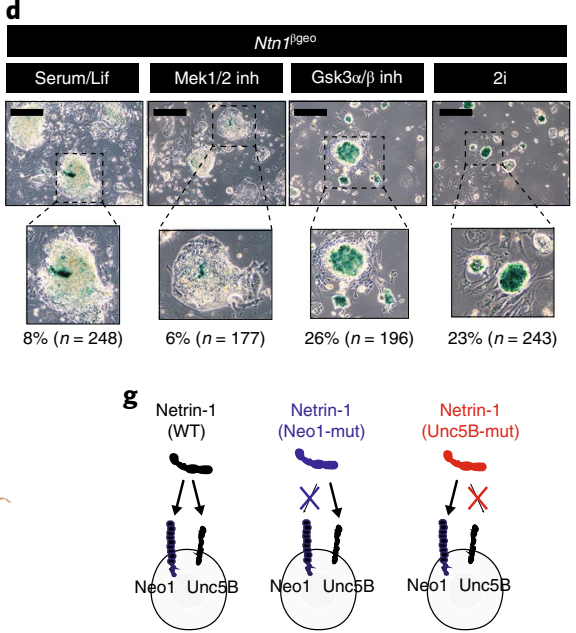

h

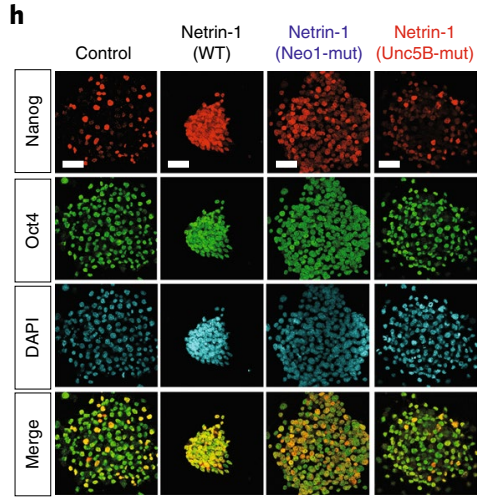

m

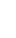
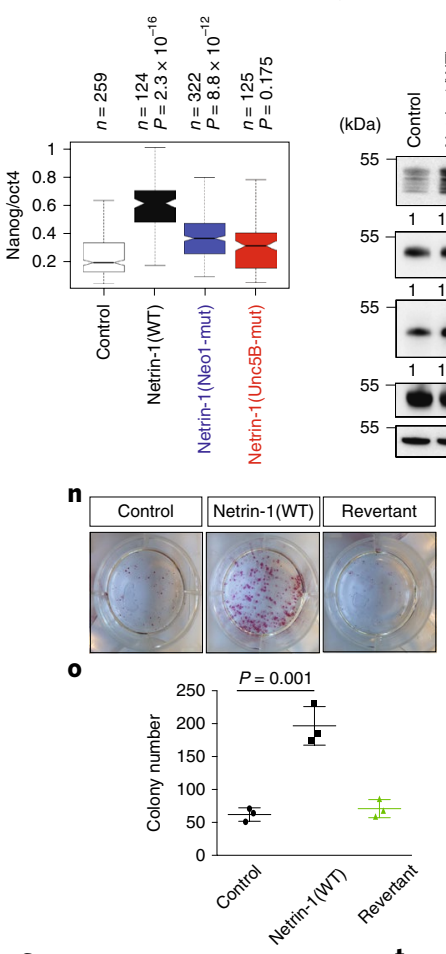

k

j

j
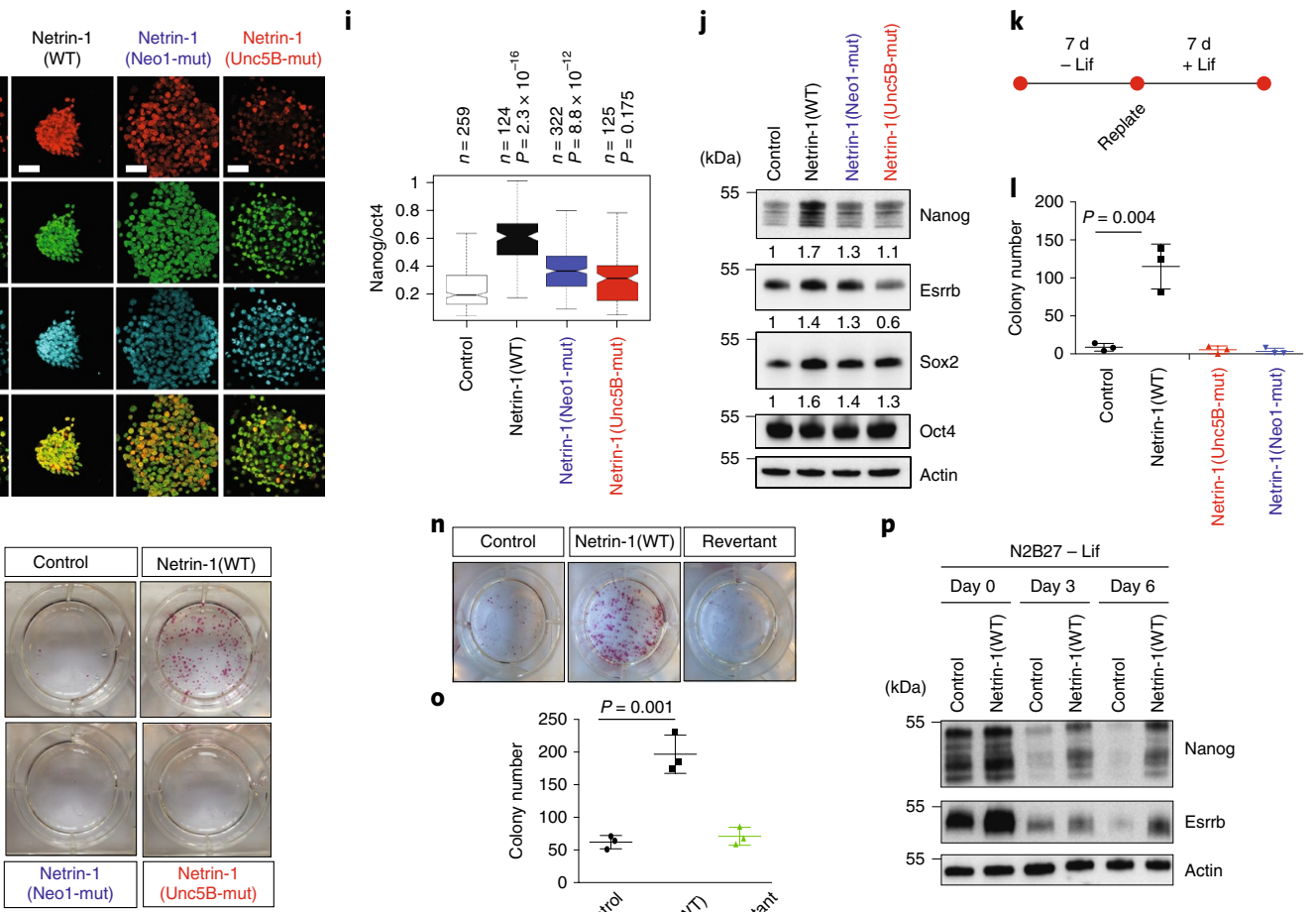

$\mathbf{q}$
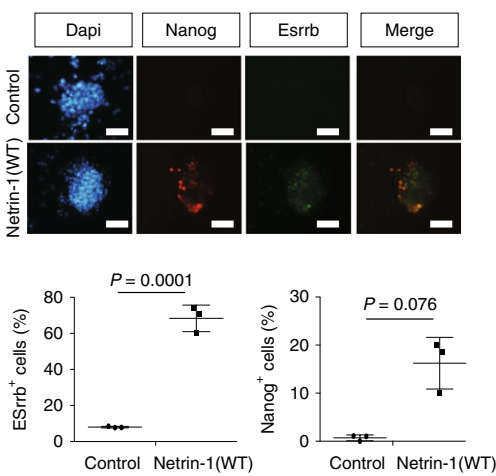

$\mathbf{S}$

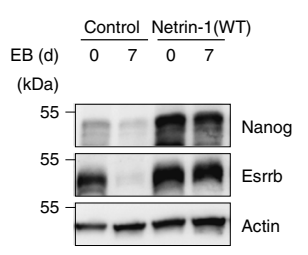

$\mathbf{t}$

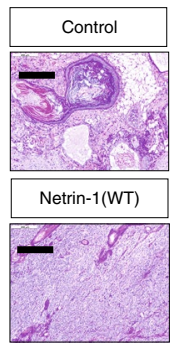

$\mathbf{p}$

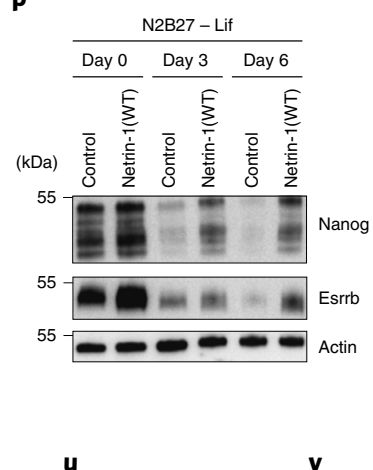

Netrin-1

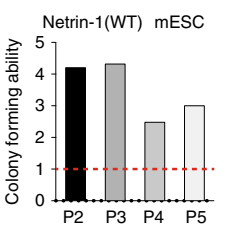

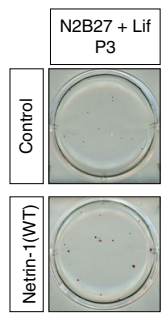


Netrin-1-Neo1-Unc5B signalling axis sustains Nanog and protects mESCs from differentiation.

The activation of the Netrin-1-Neo1-Unc5B signalling axis induces transcriptomic and epigenetic changes in mESC. To gain insight into Netrin-1 function, we compared the transcriptomes of control and Netrin-1(WT) mESCs grown in serum/Lif. Netrin1 (WT) affected expression of 434 genes (fold change $(\mathrm{FC})>1.5$ or FC $<-1.5$ at adjusted $P<0.05$ ), with strong repression of differentiation genes (Gata6 and Gata4) (Fig. 2a). Functional-annotation clustering of differentially expressed genes (DEGs) by gene ontology (GO) Panther analysis revealed association with 'embryo development', 'endoderm development' and 'regulation of MAPK cascade' (Extended Data Fig. 2k). Differences were also observed in cell proliferation, in agreement with the accelerated growth of Netrin-1(WT) mESCs (Extended Data Fig. 2l,m). Because similar GO terms are modulated with $2 \mathrm{i}^{8}$, we investigated whether Netrin-1 triggered ground-state pluripotency features. We compared the transcriptomes of control and Netrin-1(WT) mESCs grown in serum/Lif with those from published datasets ${ }^{30,31}$ (see Methods). The transcriptome of Netrin-1(WT) cells acquired limited but significant similarities with $2 \mathrm{i}$ mESCs (Fig. 2b). To evaluate the role of the receptors, we next compared the transcriptomic effects of Netrin1(WT), Netrin-1(Unc5B-mut) and Netrin-1(Neo1-mut) (Fig. 2c,d). Both mutant forms failed to induce the full transcriptomic signature, supporting the importance of the interaction of Netrin-1 with both Neo1 and Unc5B in regulation of mESC physiology.

We assessed whether Netrin-1 signalling instructs groundstate-related epigenomic modifications. We found that Netrin1 (WT) mESCs displayed increased activity of the naive Oct4 distal enhancer alongside Jarid 2 and $\operatorname{Prdm} 14$ enhancers, which were also induced with $2 \mathrm{i}^{32}$ (Fig. 2e). Chromatin immunoprecipitation with sequencing (ChIP-seq) analyses revealed a global increase in both H3K4me3 and H3K27me3 histone marks in Netrin-1(WT) mESCs (Fig. 2f), which included increased H3K4me3 enrichment at pluripotency genes Nanog and Sox2 (Fig. 2g). We also observed increases in both $\mathrm{H} 3 \mathrm{~K} 4 \mathrm{me} 3$ and $\mathrm{H} 3 \mathrm{~K} 27 \mathrm{me} 3$ at bivalent promoters ${ }^{8}$ (Fig. 2h,i). In $2 \mathrm{i}$, reduced levels of Dnmt3A, Dnmt3B, Dnmt3L and Uhrf1 trigger genome-wide DNA hypomethylation ${ }^{10,13,33,34}$. By contrast, restricted representation bisulfite sequencing revealed that Netrin1 (WT) mESCs (grown for more than 30 passsages in the presence of the transgene) display DNA methylation resembling that of control cells (mean $=0.25 \pm 0.003$ and $0.24 \pm 0.004$ respectively) (Fig. $2 j$ and Extended Data Fig. 2n). In addition, even if the level of Dnmt3A was slightly reduced (Extended Data Fig. 2o), Uhrf1 protein level was induced in Netrin-1(WT) mESCs (Fig. 2k). Moreover, even though $2 \mathrm{i}$ represses differentiation genes of the three germ layers ${ }^{8}$, ectodermal genes were not downregulated in Netrin-1(WT) mESCs (Fig. 21).
Collectively, these results indicate that the Netrin-1-Neo1-Unc5B signalling axis induces transcriptomic and epigenomic changes in mESC that share limited analogies with $2 \mathrm{i}$.

Netrin-1 controls Wnt and MAPK signalling by modulating Gsk3 $3 / \beta$ and Erk1/2 in mouse and human pluripotent stem cells. We dissected how Netrin-1 instructs pluripotency features at the molecular level. With $2 \mathrm{i}$, blockade of Gsk $3 \alpha / \beta$ activates the Wnt pathway, whereas Mek1/2 inhibition suppresses MAPK signalling ${ }^{9}$. The Wnt pathway was strongly activated in Netrin1 (WT) mESCs, as indicated by $\beta$-catenin levels, and expression of Netrin-1 mutants showed that Neo1 and Unc5B were required for this effect (Fig. 3a). Gsk3 $\alpha / \beta$ activity was also reduced in Netrin1 (WT) mESCs, as revealed by increased levels of its inactive form ${ }^{35}$ (Fig. 3a). Moreover, the level of inactive phosphorylated (p-) Gsk $3 \alpha / \beta$ in Netrin-1(WT) mESCs was equivalent to that in control cells treated with Wnt3a, and could not be increased further by Wnt3a addition (Fig. 3b).

Netrin-1 has been linked to various kinases ${ }^{36-40}$ that may be involved in Gsk3 $\alpha / \beta$ phosphorylation. Among these, we found slightly increased levels of active Fak in Netrin-1(WT) mESCs (Fig. 3c). Because Fak was shown to phosphorylate $G s k 3 \alpha / \beta^{41}$, we investigated whether the effect of Netrin-1 on Wnt is mediated by Fak. Depletion of Fak using short interfering RNA (siRNA) in Netrin-1(WT) mESCs (>80\%; Extended Data Fig. 3a) reduced p-Gsk $3 \alpha / \beta$, thereby reducing Wnt-pathway activation (Fig. 3d).

We next showed that Netrin-1(WT) mESCs harbour reduced levels of p-Erk1/2, whereas p-Mek1/2 levels remained similar to those in the control cells (Fig. 3e). Because MAPK activation is controlled by Lif and Fgf, we determined whether Netrin-1 signalling modulated the sensitivity of mESCs to these cytokines. Deprivation and stimulation experiments indicated that Fgf4 responsiveness was markedly reduced in Netrin-1(WT) mESCs, as indicated by p-Erk1/2 (Fig. 3f). In similar settings, Lif-mediated p-Erk1/2 and p-Stat3 inductions were not affected (Extended Data Fig. 3b). The link between Unc5B and the phosphatase complex $\mathrm{Pp} 2 \mathrm{a}^{36}$ prompted us to investigate whether this complex regulated the decrease in p-Erk1/2 in $\mathrm{mESCs}^{42}$. Immunoprecipitation of the catalytic subunit Pp2aco showed that Netrin-1 activation triggered a substantial increase of its phosphatase activity (Fig. $3 \mathrm{~g}$ ). The qualitative composition of the complex has been shown to modulate its activity ${ }^{43}$, and we observed an induction of the regulatory subunit ppp2r2c in Netrin-1(WT) mESCs (Fig. 3h). To evaluate whether the Pp2a complex is responsible for MAPK attenuation, we attempted to rescue p-Erk1/2 levels by siRNA-mediated depletion of Ppp2ca or Ppp2r2c. We showed that knockdown ( $>80 \%$; Extended Data Fig. 3c) of both subunits rescued p-Erk1/2 level, while levels of p-Mek1/2 and $\mathrm{p}-\mathrm{Gsk} 3 \alpha / \beta$ remained steady (Fig. 3i).

Fig. 2 | Netrin-1 signalling triggers transcriptomic and epigenetic changes in mESCs. a, Volcano plot comparing transcriptomes of control and Netrin1 (WT) mESCs. $n=3$ independent samples. Benjamini-Hochberg adjusted $P$ values of the comparisons were computed using the limma-voom workflow; modified two-sided t-test. b, Hierarchical clustering of transcriptomes with published datasets ${ }^{30,31}$ (see Methods for details). c,d, Comparison of the effects of Netrin-1(WT), Netrin-1(Unc5B-mut) (c) and Netrin-1(Neo1-mut) (d) on the transcriptome of mESCs. $n=3$ independent samples. Benjamini-Hochberg adjusted $P$ values of the comparisons were computed using the limma-voom workflow; modified two-sided $t$-test. e, Luciferase assays. Data are normalized to Renilla activity produced by a plasmid as a control of transfection efficiency and expressed as the mean \pm s.d. ( $n=4$ independent experiments). Two-sided Student's t-test. f-i, H3K4Me3 and H3K27Me3 distribution in control and Netrin-1(WT) mESCs. Data are representative of 3 independent experiments. f, H3K4Me3 and H3K27Me3 distribution in control and Netrin-1(WT) mESCs. Data are representative of 3 independent experiments. Modified two-sided $t$-test. Normalized enrichment of H3K4me3 and H3K27me3 for ENCODE-annotated embryonic stem cell peaks (ENCFF001XWU and ENCFF001XWQ, respectively) located at least $10 \mathrm{~kb}$ from bivalent promoters. $\mathbf{g}$, Representative browser tracks of H3K4me3 enrichment (in fragments per kilobase of transcript per million (FPKM)) at Nanog and Sox2 loci. h, Normalized H3K4Me3 and H3K27Me3 enrichment at bivalent promoters in control and Netrin-1(WT) mESCs. i, Representative browser tracks of H3K27me3 enrichment (FPKM) at bivalent loci. j, Heat map of methylation levels for 1.3 million matched CpGs in control, Netrin-1(WT) mESCs grown in serum/Lif and 2i mESCs. Each horizontal line is one CpG. k, Western blot for Uhrf1 in indicated mESCs grown in serum/Lif (3 independent experiments). I, Expression of differentiation-related genes in control, 2i and Netrin-1(WT) mESCs. Dendogram presents RNA-seq data. FPKM values are normalized to control mESCs and presented as $\log _{2}$ values. Colour scale is provided. 
mESCs with Netrin-1-doxi expression showed dose-dependent changes in Wnt and MAPK signalling following dox addition (Fig. 3j). When mESCs were plated on Netrin-1-doxi feeder cells, with or without dox treatment, we observed similar changes in signalling, indicating that Netrin-1 can act in a paracrine manner on pluripotency (Fig. 3k). Finally, human iPS cells exogenously expressing Netrin-1 showed limited but similar elevation of Wnt and reduction of MAPK activities-but these were not sufficient to increase Nanog expression (Fig. 31).

Collectively, these results show that activation of Netrin-1 signalling promotes Wnt signalling by activating Fak, which triggers Gsk $3 \alpha / \beta$ inactivation and $\beta$-catenin stabilization. Netrin- 1 also modifies Pp2a complex composition and activity to dephosphorylate Erk1/2.
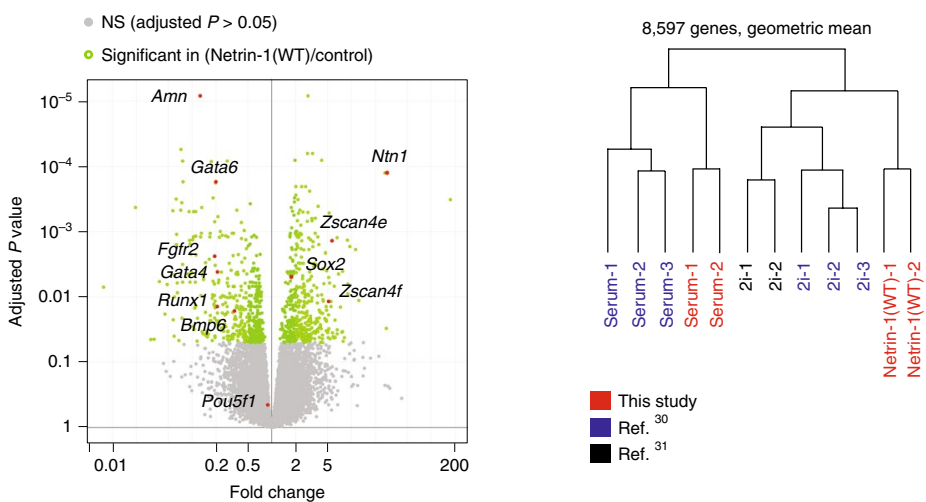

e

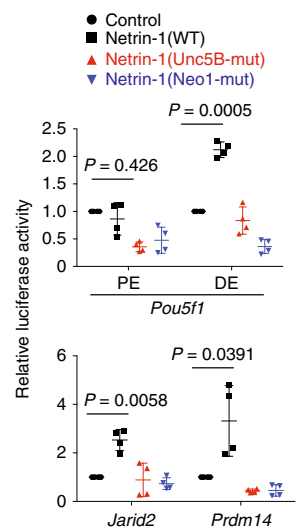

h

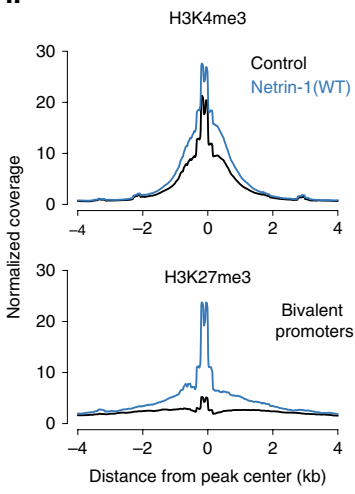

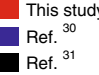

\section{f}

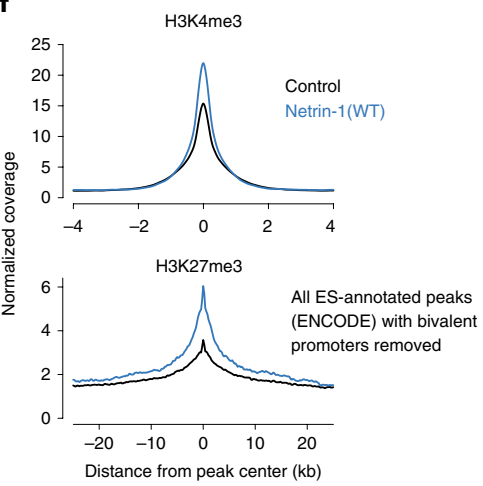

i
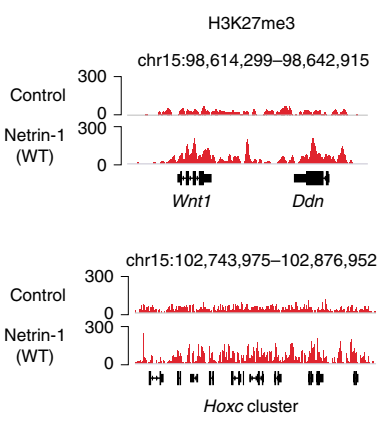

j

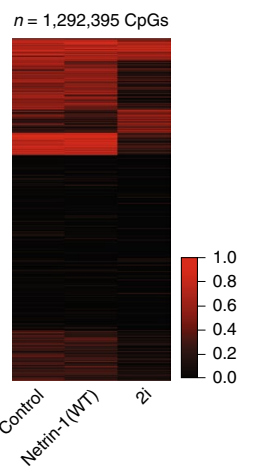

- NS (adjusted $P<0.05$

Significant (Netrin-1(WT)/control)

Significant (Netrin-1(Unc5B-mut)/control)

- Significant both

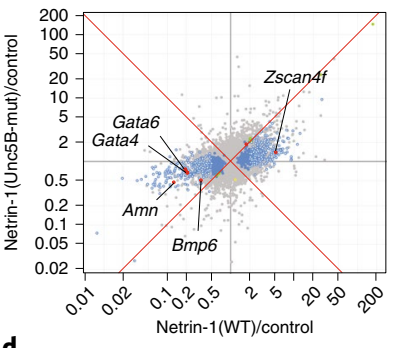

d $\quad \mathrm{NS}($ adjusted $P>0.05)$

- Significant (Netrin-1(WT)/control)

Significant (Netrin-1(Neo1-mut)/control)

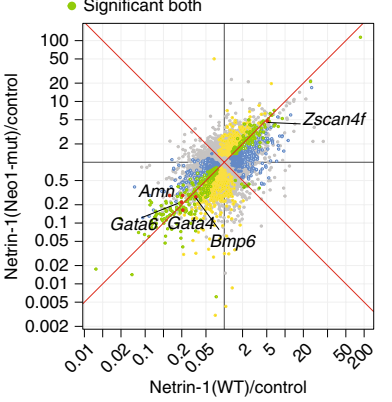

g
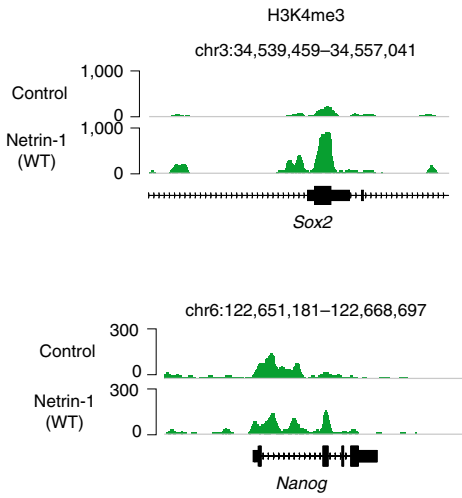

$\mathbf{k}$

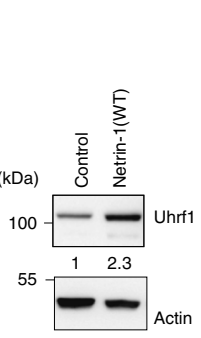

I 


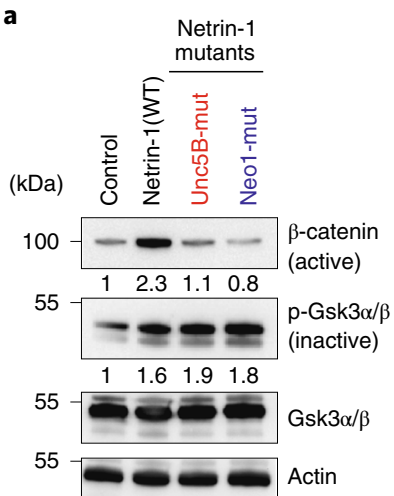

e

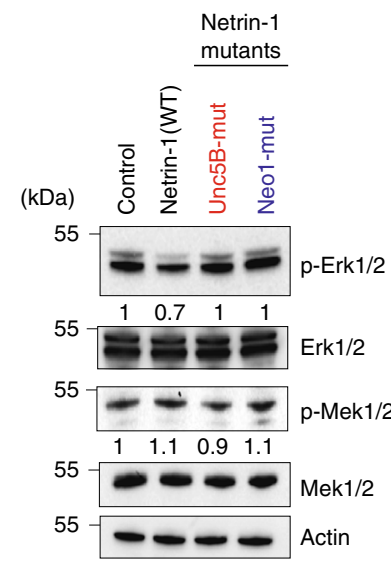

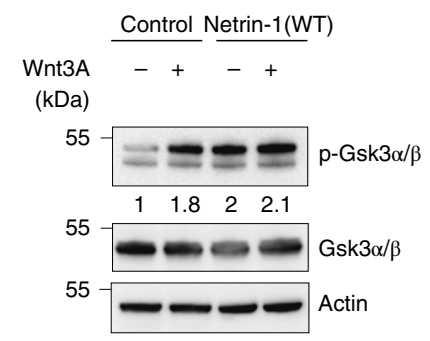

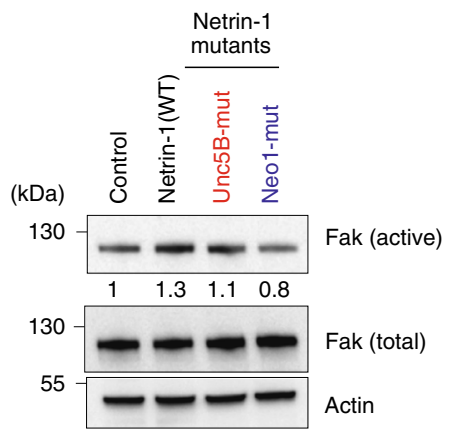

d

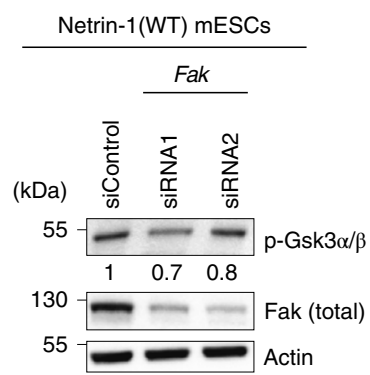

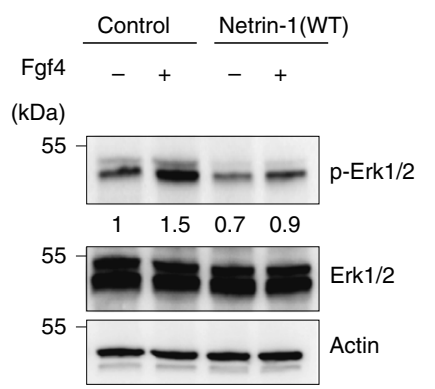

g

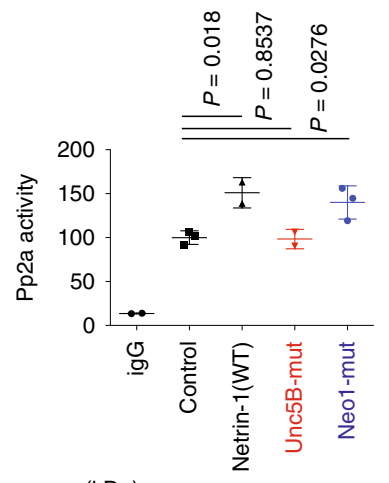

(kDa)
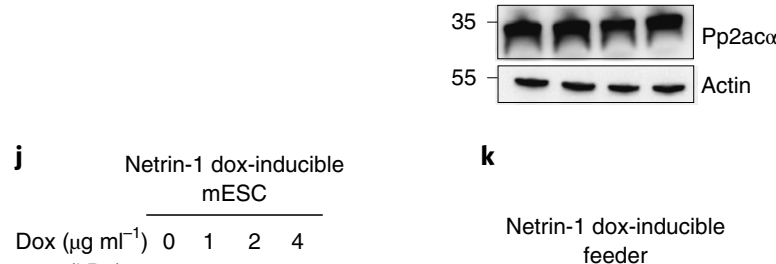

(kDa)

55

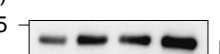
$p-G s k 3 \alpha / \beta$

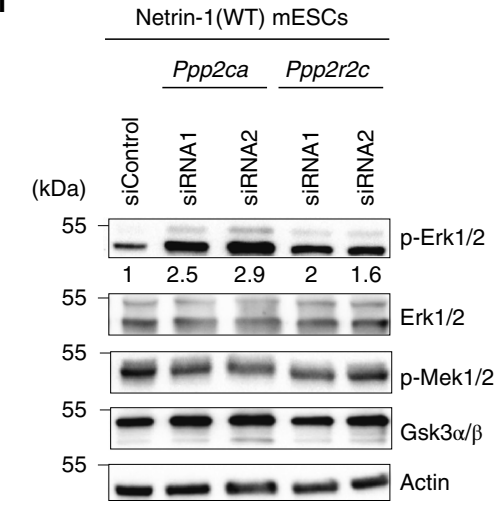

Netrin-1(WT) mESCs

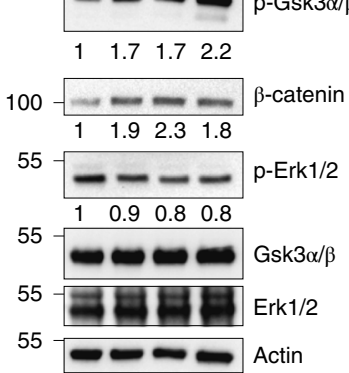

k

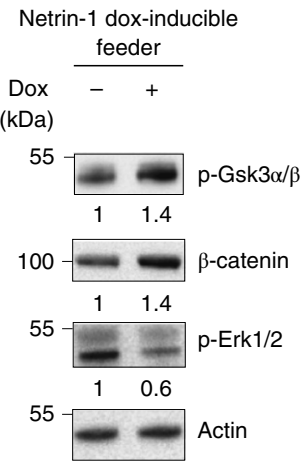

h

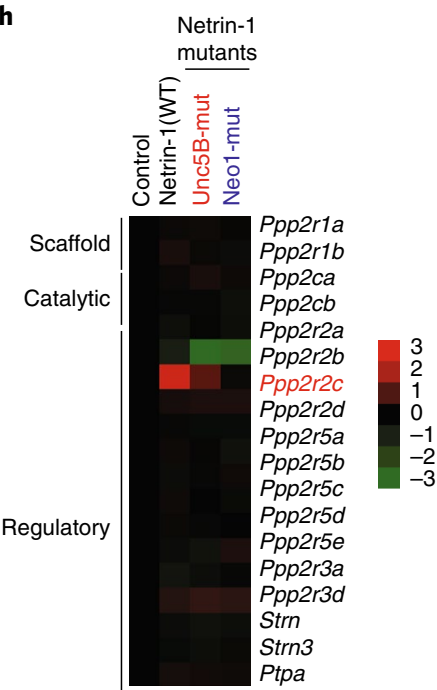

Fig. 3 | Netrin-1 regulates Gsk3 $\alpha / \boldsymbol{\beta}$ and Erk1/2 activities in mouse and human pluripotent stem cells. a, Western blot of Wnt-pathway proteins in the indicated mESCs grown in serum/Lif. b, Effect of Netrin-1 signalling on Wnt3a sensitivity. Control and Netrin-1(WT) mESCs were serum-starved overnight and stimulated with recombinant Wnt3a for $6 \mathrm{~h}$ before sample collection. c, Western blot of phosphorylated (active) and total Fak levels in control and

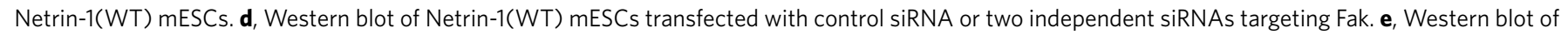
MAPK proteins in the indicated mESCs grown in serum/Lif. f, Effect of Netrin-1 on Fgf4 sensitivity. Control and Netrin-1(WT) mESCs were serum-starved overnight and stimulated with recombinant Fgf4 for 20 min before sample collection. g, Modulation of Pp2a activity by Netrin-1 signalling in mESCs. Top, phosphatase activity of the complex in Pp2aca (or control lgG) immunoprecipitates from control and Netrin-1-expressing mESCs. Data are mean \pm s.d. ( $n=3$ independent experiments). Two-sided Student's $t$-test. Bottom, western blot showing Pp2ac $\alpha$ levels in the corresponding mESC populations. $\mathbf{h}$, Dendogram showing levels of transcripts of Pp2a subunits. The raw FPKM data are normalized to control mESCs and presented as log 2 values. Three independent samples. i, Western blot in Netrin-1(WT) mESCs transfected with control siRNA or two independent siRNAs targeting Ppp2ca or Ppp2r2c. j, Western blot showing Wnt and MAPK activation in Netrin-1-doxi mESCs. Dox was added at the indicated concentrations for $48 \mathrm{~h}$. $\mathbf{k}$, Netrin-1-expressing feeder cells trigger similar signalling changes in mESCs. The irradiated feeder was plated and untreated or treated with $2 \mu \mathrm{m} \mathrm{ml}^{-1} \mathrm{dox}$ for $24 \mathrm{~h}$. The next day, mESCs were plated on the feeders and grown for $3 \mathrm{~d}$ before collection. I, Western blot of Wnt and MAPK proteins in control and Netrin-1(WT) human iPS cells. Western blots in $\mathbf{a}-\mathbf{g}$ and $\mathbf{i}-\mathbf{I}$ are representative of 3 independent experiments. 


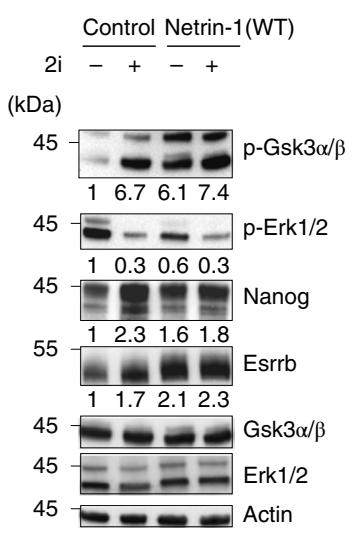

b

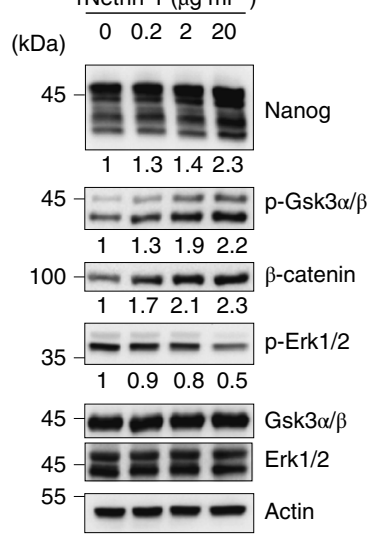

c

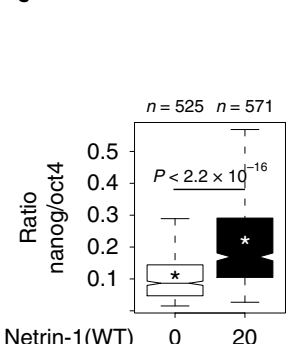

Netrin-1(WT) $0 \quad 20$ d

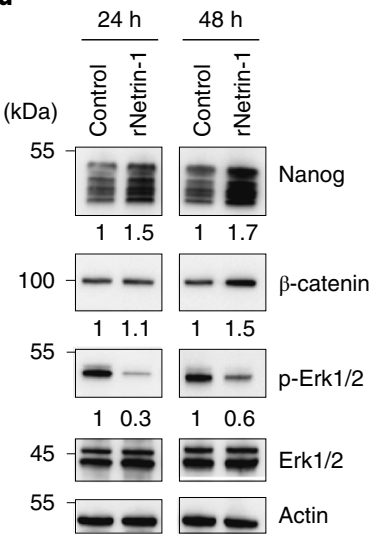

e

$24 \mathrm{~h} \log _{2} \mathrm{FC}>1$ or $<-1$; adjusted $P<0.05$

- $48 \mathrm{~h} \log _{2} \mathrm{FC}>1$ or $<-1$; adjusted $P<0.05$

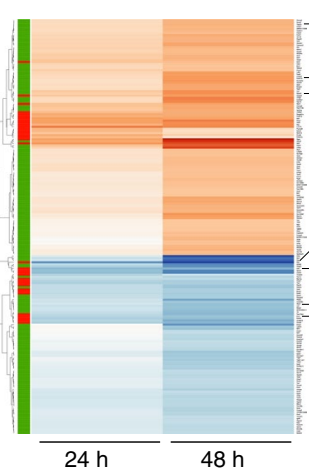

Tfap2c

Prdm14 Dppa3

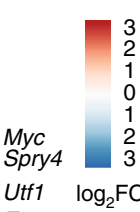

Etv4 f

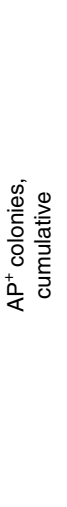

h

g

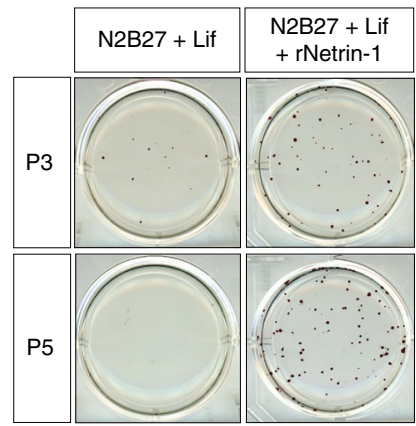

mESCs replated after 5 passages

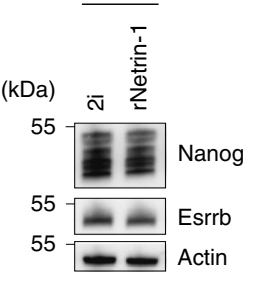

Fig. 4 | Recombinant Netrin-1 supports mESC self-renewal in combination with Lif. a, Western blot comparing signalling and pluripotency changes induced by Netrin-1 and 2i. Control and Netrin-1(WT) mESCs were grown in serum/Lif and treated with $2 \mathrm{i}$ for $2 \mathrm{~d}$ ( 3 independent experiments). b, Western blot of Nanog, Wnt and MAPK proteins in response to increasing doses of rNetrin-1. Cells were treated with the indicated concentrations for $48 \mathrm{~h}$ (3 independent experiments). c, Quantification of the Nanog/Oct4 intensity from immunofluorescence in single cells from the different populations. $n$ corresponds to the number of cells. The centre line represents the median of the data, box edges show the upper and lower quartiles and whiskers show the highest and lowest values, excluding outliers. Two-tailed Student's $t$-test. d, Western blot of pluripotency and signalling changes occurring after 24 and $48 \mathrm{~h}$ of treatment with $20 \mu \mathrm{g} \mathrm{ml}^{-1} \mathrm{rNetrin}-1$ (3 independent experiments). e, Heat map of DEGs. RNA-seq was performed on untreated or $\mathrm{rNetrin-1-treated} \mathrm{mESCs.} n=4$ independent samples. $P$ values from two-sided Wald test; two-sided Benjamini-Hochberg test for adjustment. f, Self-renewal assays. E14Tg2a mESCs were maintained for six passages in the indicated conditions. After splitting at P2, P3, P5 and P6, cells were counted and similar cell numbers were plated at clonal density in serum/Lif for $7 \mathrm{~d}$ and the number of alkaline phosphatase-positive colonies was counted to evaluate the self-renewal potential of the cells. Data from one representative experiment of two independent experiments. $\mathbf{g}$, Self-renewal abilities of mESCs grown in the indicated conditions for three or five passages. Similar results were obtained from 3 independent experiments. $\mathbf{h}$, Western blot of pluripotency factors. $\mathrm{mESC}$ cultured in N2B27 + Lif $+2 \mathrm{i}$ or $\mathrm{N} 2 \mathrm{~B} 27$ + Lif + rNetrin-1 for 5 passages (15d) were cultured in serum/Lif for a further $7 \mathrm{~d}$ before collection (3 independent experiments).

Netrin-1 supports mESC self-renewal in combination with Lif. To compare the magnitude of the changes induced by Netrin-1 and $2 \mathrm{i}$, control and Netrin-1(WT) mESCs were treated with $2 \mathrm{i}$ for $48 \mathrm{~h}$ in serum/Lif (Fig. 4a). Basal levels of p-Gsk $3 \alpha / \beta$, Nanog and Esrrb in Netrin-1(WT) mESCs were similar to those in 2i-treated mESCs. However, there was less reduction in MAPK signalling in the Netrin-1(WT) cells (Fig. 4a), and these cells remained responsive to $2 \mathrm{i}$ treatment, confirming that Netrin-1 only partially mimics signalling changes induced by $2 \mathrm{i}$.

We assessed whether recombinant Netrin-1 (rNetrin-1; see Methods) triggers similar changes to transgenes in mESCs. Using western blot and immunofluorescence, we showed that $48 \mathrm{~h}$ treatment with different doses of rNetrin-1 led to Nanog induction, demonstrating a paracrine effect of rNetrin-1 (Fig. $4 b, c)$. This treatment also led to dose-dependent changes of $\beta$-catenin, $\mathrm{p}-\mathrm{Gsk} 3 \alpha / \beta$ and p-Erk1/2, as expected (Fig. 4b). We next dissected the sequence of events by analysing mESCs at 24 and $48 \mathrm{~h}$ of treatment. Changes in signalling appeared with different kinetics: Erk1/2 phosphorylation was decreased at $24 \mathrm{~h}$, whereas $\beta$-catenin was induced only at $48 \mathrm{~h}$ (Fig. 4d). Transcriptomic analyses enabled us to define clusters of early and late responders (Fig. $4 \mathrm{e}$ ). At $24 \mathrm{~h}$, there was a very limited response $\left(35\right.$ DEGs, $\log _{2}$ FC $<1$ or $>1$ and adjusted $P<0.05$ ) and significant downregulation was detected for pluripotency genes (Myc and Utf1). Differentiation genes (Hand1) and Fgf targets (Etv4, Spry4 and Dusp6) were also repressed, in line with the rapid changes of $\mathrm{p}$-Erk1/2. At $48 \mathrm{~h}$, a larger transcriptomic response was evident (193 DEGs), including upregulation of Tfap2c, Prdm14 and Dppa3. Most of the endoderm and mesoderm genes repressed in Netrin-1(WT) mESCs (Fig. 2l) were not repressed at these early time points, suggesting that Netrin-1 induces signalling changes prior to repressing differentiation.

We next investigated whether rNetrin-1 can support mESC self-renewal in combination with Lif. mESCs were grown at clonal density on laminin in N2B27 + Lif with rNetrin-1 or $2 \mathrm{i}$ for six passages (18d) (Fig. 4f,g). Colony-formation assays confirmed that Lif is not sufficient to maintain self-renewal in serum-free 

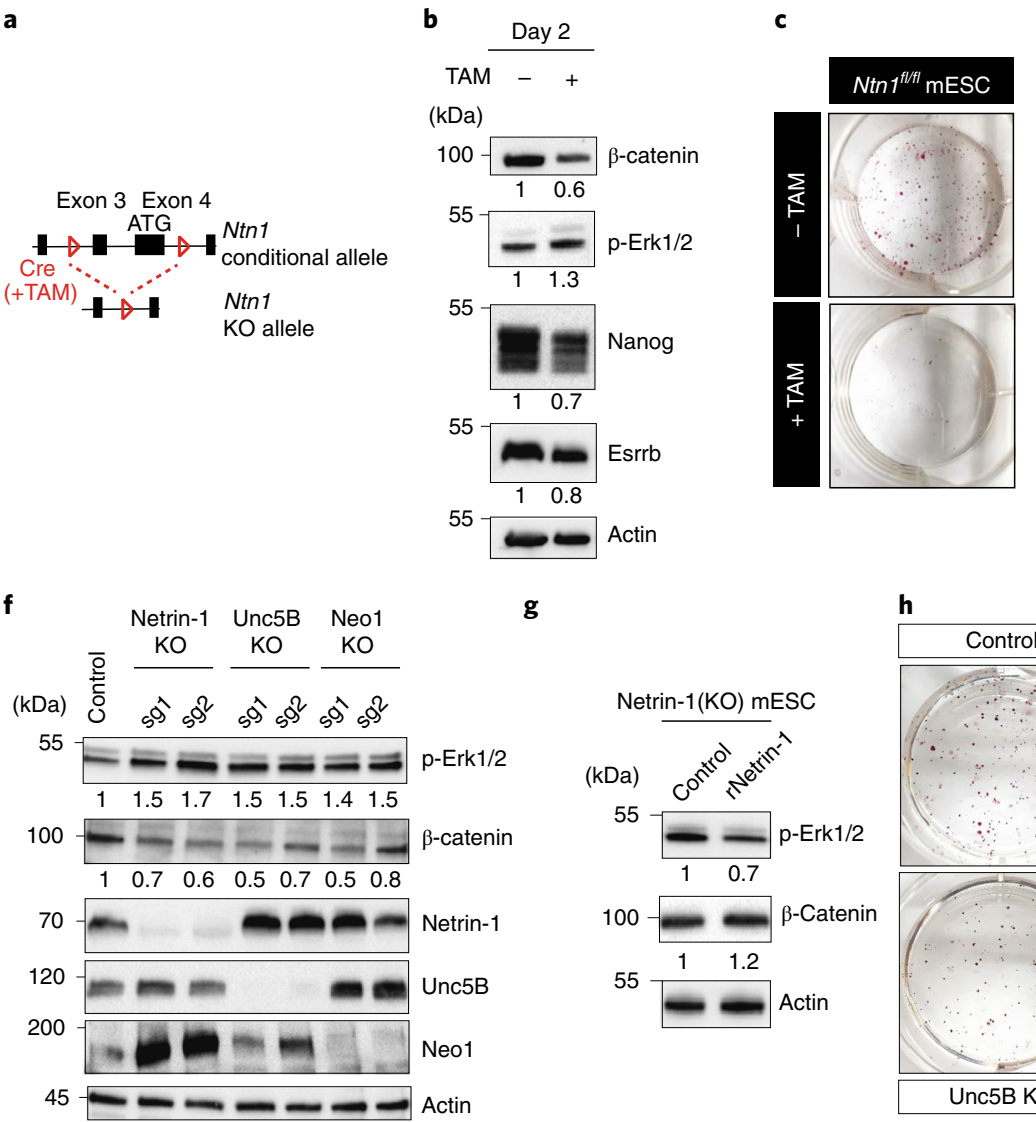

d

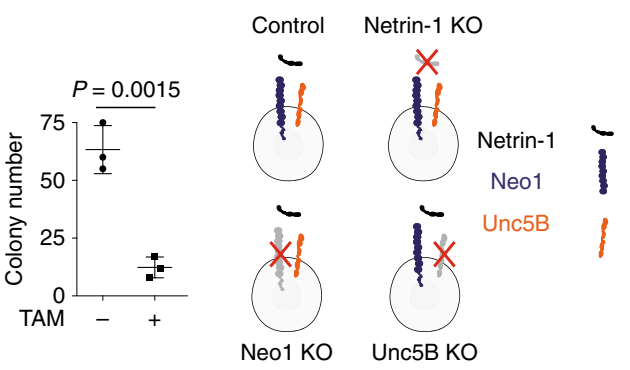

Fig. 5 | Endogenous Netrin-1 controls pluripotent features via Neo1 and Unc5b. a, Schematic of the Ntn1 conditional allele. KO, knockout. b, Western blot for Wnt and MAPK proteins in Ntn $T^{t / 1 / f l}$ mESCs treated overnight or not with tamoxifen (TAM) in N2B27 + Lif for $48 \mathrm{~h}$ before collection ( 3 independent experiments). c, Colony-formation assays. Similar results were obtained from 3 independent experiments. $\mathbf{d}$, Colony counts. Data are mean $\pm s$.d., $n=3$ independent experiments; two-sided Student's t-test. e, Scheme depicting the Netrin-1(KO), Neol(KO) and Unc5B(KO) mESCs generated using CRISPRCas9. f, Effect of Netrin-1, Neo1 and Unc5B depletion on Wnt and MAPK pathways (3 independent experiments). $\mathbf{g}$, Western blot for Wnt and MAPK proteins. Netrin-1(KO) mESCs were treated for $48 \mathrm{~h}$ with rNetrin-1 $\left(20 \mu \mathrm{g} \mathrm{ml}^{-1}\right)$ before collection (3 independent experiments). $\mathbf{h}, \mathbf{i}$, Effect of ligand or receptor depletion on $\mathrm{mESC}$ self-renewal ability. Cell lines from e were analysed by colony-formation assay. $\mathbf{h}$, Bright-field images of a single experiment representative of 3 independent experiments. $\mathbf{i}$, Colony counts. Data are mean \pm s.d., $n=3$ independent experiments; two-sided Student's $t$-test.

medium (Fig. 4f,g). However, the addition of rNetrin-1 enabled sustained self-renewal, similar to $2 \mathrm{i}$ (Fig. $4 \mathrm{f}, \mathrm{g}$ ). When replated in serum/Lif after five passages in N2B27+ Lif + rNetrin-1 or $\mathrm{N} 2 \mathrm{~B} 27+\mathrm{Lif}+2 \mathrm{i}, \mathrm{mESCs}$ exhibited similar Nanog and Esrrb levels (Fig. 4h). Together, these results show that rNetrin-1 co-regulates Wnt and MAPK signalling and sustains self-renewal in combination with Lif.

The Neo1 and Unc5b receptors are required for endogenous Netrin-1 function in mESCs. Because Netrin-1 is expressed at basal levels in serum/Lif (Fig. 1b), we assessed its endogenous function in mESCs. We generated Ntn1 conditional-knockout (KO)

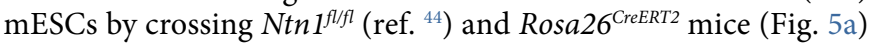
(Extended Data Fig. 4a). Because feeder cells secrete Netrin-1, $N t n 1^{f l f l}$ mESCs were adapted on gelatin. After $48 \mathrm{~h}, \mathrm{Ntn} 1$ deletion induced changes in signalling and pluripotency that mainly mirrored gain of function. Wnt-pathway activation was reduced, whereas MAPK activity was induced and Nanog and Esrrb levels were reduced (Fig. 5b). However, the expression of epiblast ( Fgf5 and Otx2) or primitive endoderm (Gata4 and Gata6) transcripts was not significantly induced (Extended Data Fig. 4b). Netrin-1 loss also led to a significant decrease of mESC self-renewal ability (Fig. $5 c, d$ ) with no significant changes in proliferation and cell death (Extended Data Fig. 4c,d). These defects, observed in the first days following Netrin-1 deletion, were compensated and $N t n 1^{-1-}$ mESCs could be maintained at high density for more than 20 passages (Extended Data Fig. 4e).

We next generated Netrin-1-, Neo1- and Unc5B-KO mESCs using clustered regularly interspaced short palindromic repeats (CRISPR)-CRISPR-associated protein 9 (Cas9) genome editing (Fig. 5e,f and Extended Data Fig. 4f). CRISPR-Cas9-mediated loss of Netrin-1 led to similar changes in p-Erk1/2 and $\beta$-catenin levels as the conditional $\mathrm{KO}$ (Fig. 5f), and these changes were partially rescued by treatment with rNetrin-1 (Fig. $5 \mathrm{~g}$ ). Loss of Netrin-1 triggered a self-renewal defect (Fig. 5h,i) that was compensated in $2 \mathrm{i}$ (Extended Data Fig. 4g), in agreement with the effects of Netrin-1 on Wnt and MAPK. Importantly, in serum/Lif, Unc5B and Neo1 single-KO induced signalling and clonogenicity changes that largely mimicked loss of Netrin-1 (Fig. $5 f-i$ ), indicating that a tight regulation of receptor dosage is required to co-regulate Wnt-MAPK and therefore self-renewal.

Netrin-1 regulates cell-fate allocation in preimplantation embryos. Netrin-1 depletion has been reported to cause embryonic lethality at embryonic day (E)14.5 (ref. ${ }^{45}$ ), but no function has been reported in preimplantation embryos. Due to the unexpected function we described in mESCs, we assessed whether Netrin-1 could be expressed and have a role at an earlier stage. In situ hybridization 


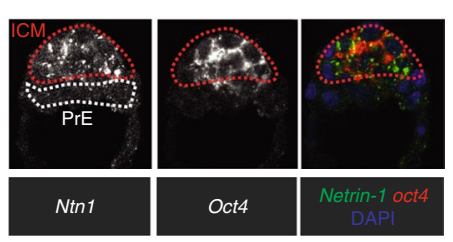

b

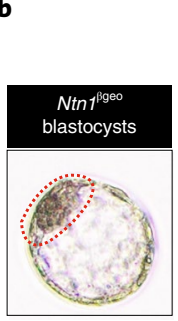

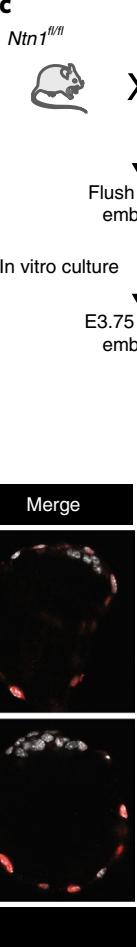

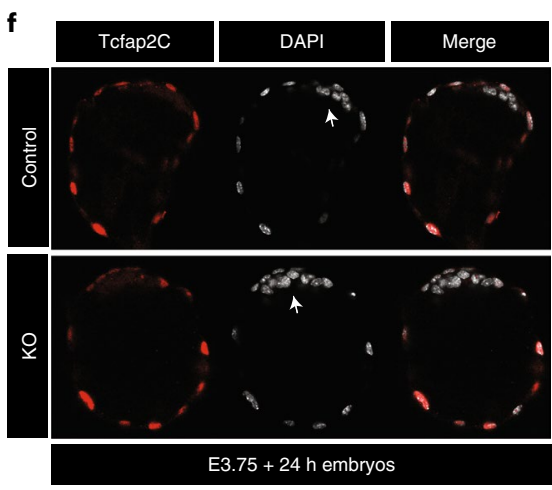

g

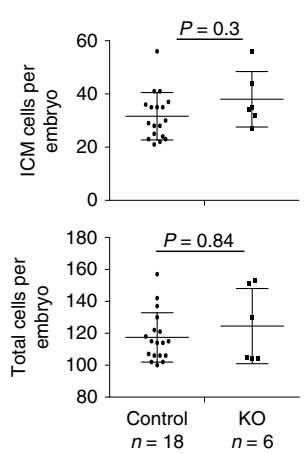

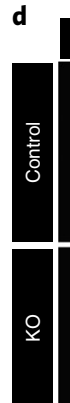

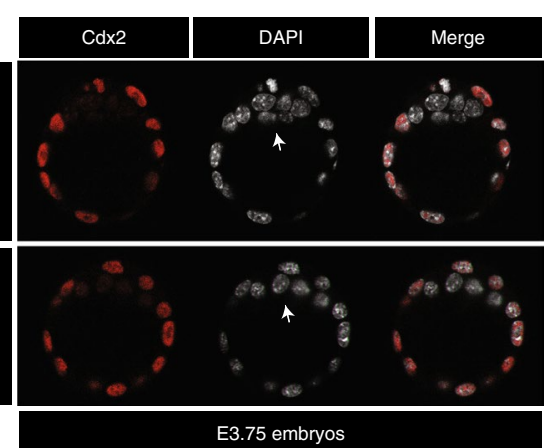

e

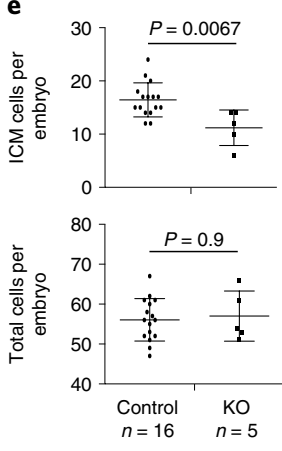

h

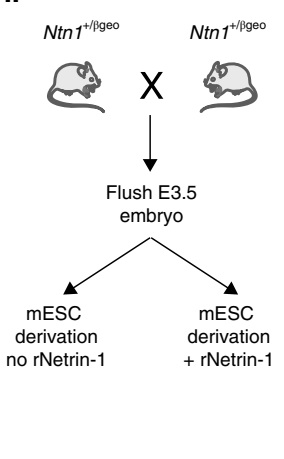

i

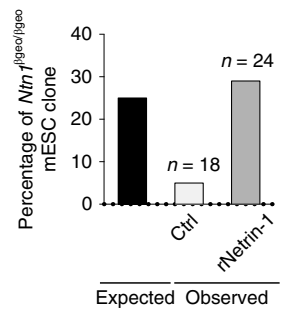

Fig. 6 | Netrin-1 regulates cell-fate allocation in preimplantation embryos. a, In situ hybridization of Netrin-1 and Oct4 in an E4.5 embryo. Representative of 17 E4.5-E4.75 embryos from 3 independent experiments. ICM, inner cell mass; PrE, primitive endoderm. $\mathbf{b}$, X-gal activity in blastocyst-stage embryos.

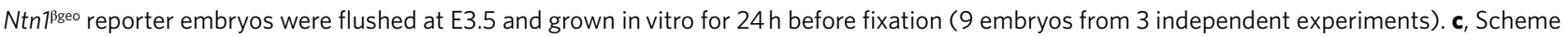
depicting the intercrosses. d, Image of control and Netrin-1(KO) E3.75 embryos. Cdx2 immunofluorescence marks trophectoderm cells. Arrows indicate ICM cells ( 4 independent experiments). e, Average number of ICM cells per embryo. Data are mean \pm s.d. of (DAPI+ cells) $-\left(C \mathrm{Cdx} 2^{+}\right.$cells); 16 control and

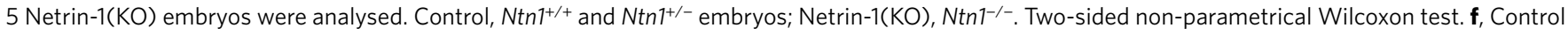
and Netrin-1(KO) E3.75 embryos grown in vitro for a further 24h. Immunofluorescence for Tcfap2C marks trophectoderm cells. Arrows indicate epiblast cells ( 3 independent experiments). $\mathbf{g}$, Number of ICM cells per embryo. Data are mean \pm s.d. of (DAPI ${ }^{+}$cells) $-\left(\right.$Tcfap $2 \mathrm{c}^{+}$cells). $n=18$ (control) and 6 (Netrin-1(KO)) embryos. Two-sided non-parametrical Wilcoxon test. h, Scheme of the intercrosses. $\mathbf{i}$, The percentage of Ntn $7^{\beta \text { geo/pgeo }} \mathrm{mESC}$ lines obtained from $\mathrm{Ntn} 1^{+/ \beta g e o}$ heterozygous crosses. $n$ represents the number of lines generated and is indicated on the graph. Embryos were flushed at E3.5, grown on feeder cells in N2B27 + Lif + 2i for 3 d and then in serum/Lif. Netrin-1 status was evaluated by western blot.

of Ntn1 and Oct4 revealed that Ntn1 expression was confined to the naive epiblast, while no signal was detected in the adjacent primitive endoderm (Fig. 6a). Previously published single-cell transcriptomic data confirmed significantly higher Ntn1 expression in epiblastic cells than in primitive endoderm in E4.5 embryos ${ }^{46}$ (Extended Data Fig. 4h). The use of $N t n 1^{\text {Bgeo }}$ embryos also showed specific $\beta$ gal activity in the epiblast of blastocysts, indicating that Netrin-1 is expressed in pluripotent blastomeres (Fig. 6b).

We next analysed E3.75 and E4.5 (E3.75 grown in vitro for 24h) embryos from intercrosses between $N \operatorname{tn} 1^{f / f l}$ males and $N t n 1^{f / \mathrm{WT}}$; EIIa-cre ${ }^{+/-}$females (Fig. 6c). This strategy was selected because offsprings inheriting EIIa-cre maternally exhibit a widespread reporter expression ${ }^{47}$. Netrin-1 depletion led to a significant reduction of the number of cells in the inner cell mass $\left(\mathrm{DAPI}^{+} \mathrm{Cdx} 2^{-}\right)$in E3.75 embryos, indicating a function for Netrin-1 in the homeostasis of the inner cell mass (Fig. 6d,e). This defect was compensated when embryos were grown in vitro and analysed $24 \mathrm{~h}$ later, in agreement with a lack of requirement for Netrin-1 at these embryonic stages (Fig. 6f,g).

We finally assessed whether Netrin-1 controls mESC derivation. Starting from blastocysts obtained through $N t n 1^{+/ \beta g e o}$ mice crosses $^{27}$ (Fig. 6h), 18 expanded blastocyst outgrowths were subsequently amplified. Among these, a single Ntn $1^{\text {Bgeo//geo }} \mathrm{mESC}$ line was detected (Fig. 6i), a deviation from the expected 1:2:1 genotype frequency. The use of rNetrin-1 rescued this deviation (Fig. 6i), indicating that Netrin-1 controls optimal pluripotency capture. Together, these results reveal an unexpected function for Netrin-1 during preimplantation development.

Netrin-1 exerts different effects in mESCs depending on the Neo1/Unc5B stoichiometry. Netrin-1 has been shown to trigger opposite responses depending on the receptor dosage ${ }^{21-23}$. We investigated whether it also exerts different functions depending on the Neo1/Unc5B stoichiometry in mESCs and differentiated derivatives. While Netrin-1 and both receptors are expressed in mESCs, embryoid body formation induced Netrin-1 expression and tilted the dosage toward a $\mathrm{Neo} 1^{\text {low }} / \mathrm{Unc} 5 \mathrm{~B}^{\text {high }}$ ratio (Fig. $7 \mathrm{a}$ ). Unc5b induction was already detected in epiblast-like (EpiLC) cells ${ }^{48}$ (Extended Data Fig. 5a). To alter experimentally the ligand and receptors ratio, we engineered control and Netrin-1(WT) mESCs to express Neo1 or Unc5B on addition of dox (Fig. 7b). We found that the decrease in $\mathrm{p}$-Erk1/2 triggered by Netrin-1 was more pronounced when Neo1 was exogenously expressed (Fig. 7c). By contrast, when Unc5B was induced, p-Erk1/2 levels were increased by Netrin-1, indicating that the ligand has different effects on MAPK depending on the balance of its receptors (Fig. $7 \mathrm{~d}$ ). In line with a function of $\mathrm{p}$-Erk1/2 in lineage commitment, we found that this setting, Netrin- $1^{\text {high }} \mathrm{Neo} 1^{\text {low } U n c 5} \mathrm{~B}^{\text {high }}$, triggered downregulation of Nanog 


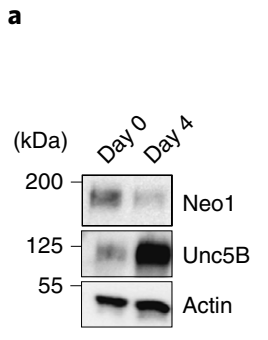

e

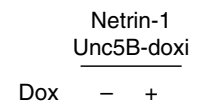

(kDa)

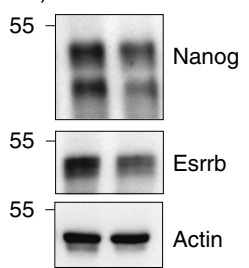

b

잉
Control mESC

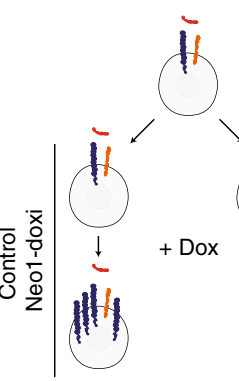

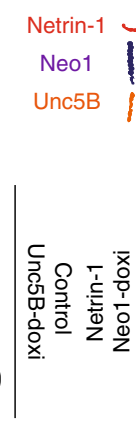

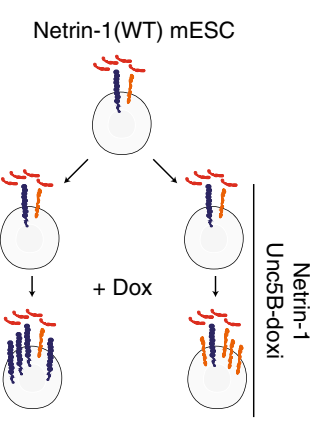

c
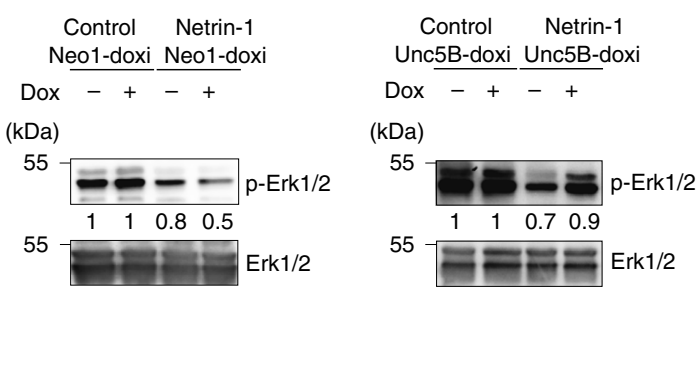

i

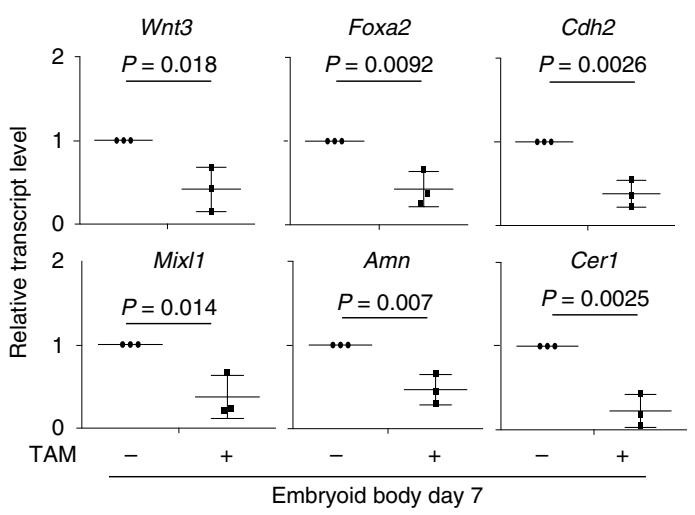

n

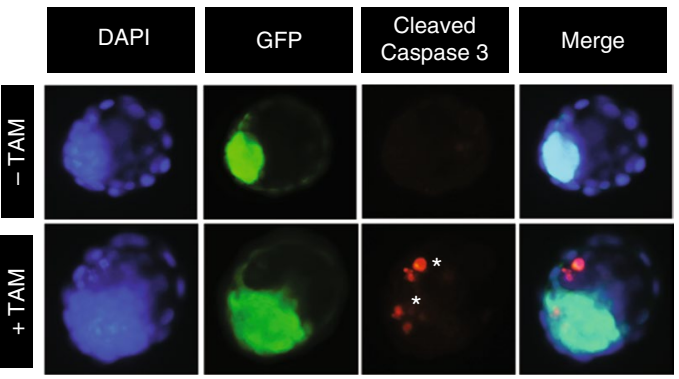

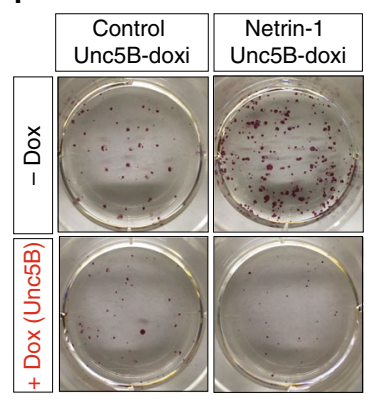

g

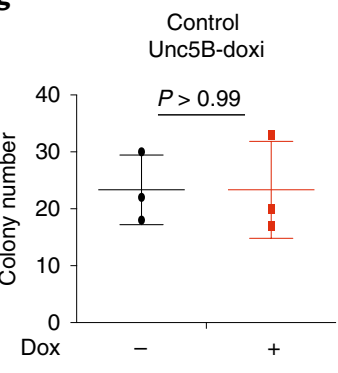

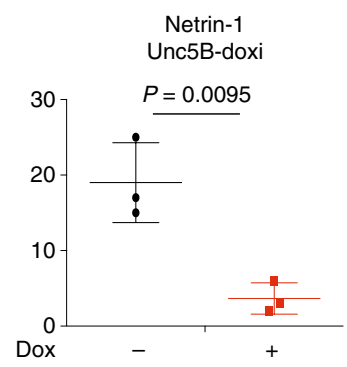

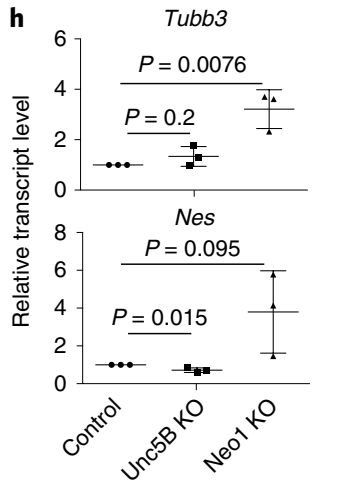

j

k
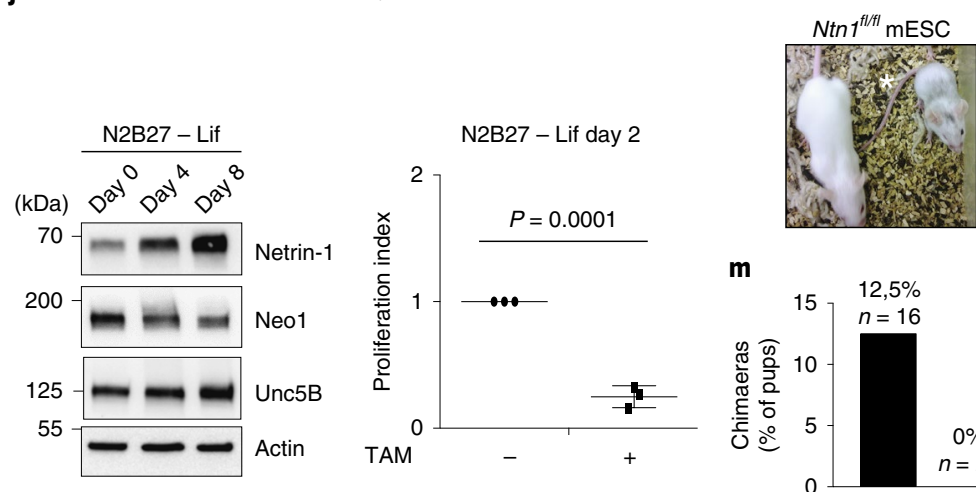

m
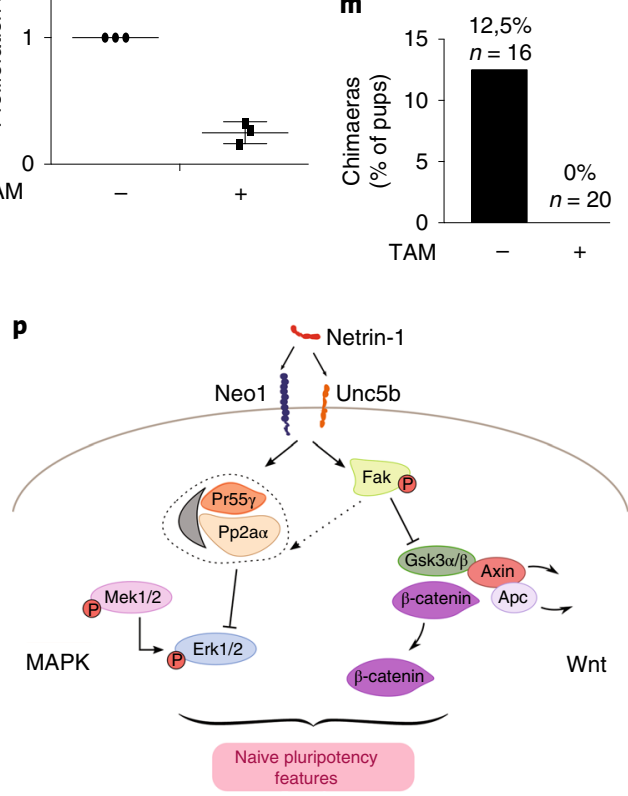

and Esrrb (Fig. 7e) and a reduction of mESC resistance to differentiation (Fig. $7 \mathrm{f}, \mathrm{g})$. In addition, by subjecting mESCs expressing solely Unc5B (Neol(KO)) or Neol (Unc5B(KO)) to differentiation in N2B27 - Lif, we found that mESCs expressing Unc5B exhibited increased induction of differentiation genes (Fig. 7h). Collectively, these data indicate that the effect of Netrin-1 on self-renewal is tightly regulated by the balance of Netrin-1 receptors.
Netrin-1 coordinates differentiation in vitro and in vivo. Loss of a pro-self-renewal signal in mESCs leads to their accelerated differentiation, as shown for $\mathrm{Lif}^{4}, \mathrm{Wnt}^{49}$ and $\mathrm{Bmp}^{6}$. However, because Netrin-1 can either repress or induce p-Erk1/2, we assessed whether and how its loss affects mESC differentiation. We subjected $N t n 1^{f l / f l}$ mESCs, untreated or treated with TAM, to differentiation assays. In embryoid bodies, Netrin-1 deletion led to a delay in the induction 
Fig. 7 | Netrin-1 exerts different effects in mESCs depending on receptor balance. a, Western blot in mESCs and day 4 embryoid bodies. b, Schematic of $\mathrm{mESC}$ lines generation. c-e, Western blot of $\mathrm{p}$-Erk1/2 levels in indicated cell lines. $\mathbf{f}, \mathbf{g}$, Assays for exit from pluripotency performed on the indicated cell lines. $\mathbf{f}$, Representative images of a single experiment representative of 3 independent experiments. $\mathbf{g}$, Colony counting. Data are mean \pm s.d. ( $n=3$ independent experiments). Two-sided Student's t-test. $\mathbf{h}$, Results of quantitative PCR with reverse transcription (RT-qPCR), showing transcript levels at day 8 of differentiation in N2B27 - Lif. Data are normalized to housekeeping genes and value 1 is given to day 8 control mESCs. Data are mean \pm s.d. (3 independent experiments). Two-sided Student's t-test. i, RT-qPCR showing levels of differentiation transcripts in day 7 embryoid bodies generated with Ntn $\mathrm{fl}^{\mathrm{f} / \mathrm{fl}} \mathrm{mESC}$, untreated or treated with TAM. Data are mean \pm s.d., $n=3$ independent experiments; value 1 is given to untreated mESCs. Twosided Student's t-test. j. Western blot showing Netrin-1, Unc5b and Neo1 expression during neural differentiation. $\mathbf{k}$, Proliferation assays. Ntn ${ }^{1 / 1 / 1}$ mESCs, untreated or treated with TAM, were grown for $2 \mathrm{~d}$ in N2B27 - Lif and cell number was counted. Data are mean \pm s.d., 3 independent experiments; twosided Student's t-test. I, m, Blastocyst injections performed with Ntn $7^{f / f l l} \mathrm{mESC}$, untreated or treated with TAM prior to injection. I, Example of a coat colour chimaera obtained following injection of $N \operatorname{tn} 7^{f / f / l} \mathrm{mESC}$. $\mathbf{m}$, Percentage of chimaeras obtained ( $n$ represents the number of live pups obtained and is indicated on the graph). $\mathbf{n}$, Image of representative embryos immunostained for GFP and cleaved Caspase-3. Morulas are aggregated with 5-10 Ntn $7^{1 / / f l}$ mESCs, untreated or treated with TAM. Blastocysts were fixed $36 \mathrm{~h}$ after aggregation. Asterisks mark apoptotic cells. $\mathbf{0}$, Count of cells positive for cleaved Caspase-3. Data are mean \pm s.d., 3 independent experiments; $n$ represents the number of embryos analysed and is indicated on the graph. Two-sided Student's t-test. p, Graphical summary of the results. Western blots in $\mathbf{a}, \mathbf{c}-\mathbf{e}, \mathbf{j}$, are representative of 3 independent experiments.

of differentiation genes of the three germ layers (Fig. 7i), indicating that Netrin-1 contributes to coordinated differentiation. Cells depleted of Netrin-1 generated teratomas of similar size to those generated by control cells (Extended Data Fig. 5b). To assess whether these defects were associated with differentiation, proliferation and/or cell death, we performed guided neural differentiation, during which Netrin-1 is induced and the switch of receptors occurs (Fig. 7j). After $8 \mathrm{~d}$, we observed a reduced induction of differentiation transcripts in the absence of Netrin-1 (Extended Data Fig. 5c). This difference was accompanied by a reduction of proliferation, which was observed as early as day two (Fig. 7k), without significant difference in cell death (Extended Data Fig. 5d), indicating that Netrin-1 controls differentiation and proliferation. When injected into blastocysts, mESCs depleted of Netrin-1 harboured a reduced ability to give rise to coat-colour chimaeras (Fig. 7l,m). To better characterize this defect, GFP-labelled Ntn $1^{f l / f l}$ mESCs, untreated or treated with TAM, were aggregated with morulas. Immunofluorescence analysis of late blastocysts for GFP and cleaved Caspase- 3 revealed an increase in the number of apoptotic cells in embryos injected with Netrin-1-depleted cells, suggesting that Netrin-1 promotes cell survival (Fig. 7n,o). Altogether, these results demonstrate that Netrin-1 deregulation triggers differentiation, proliferation and survival defects.

\section{Discussion}

In this study, we document that the neuronal guidance cue Netrin-1, which is expressed in the epiblast and in mESCs, is an autocrine and paracrine factor that promotes pluripotent features. Netrin-1 controls self-renewal of mESCs and triggers signalling, transcriptomic and epigenetic features that partially overlap with the ground state of pluripotency ${ }^{9}$ (Fig. 7p). Even if Netrin-1 signalling acts by reducing MAPK and promoting Wnt in a similar manner to 2i, it targets a different effector of the MAPK pathway-namely Erk1/2-via Pp2a. In line with this difference and in contrast to prolonged Mek1/2 blockade, we did not observe global DNA hypomethylation in Netrin-1(WT) mESCs ${ }^{13}$. Because the use of a Src inhibitor preserves mESCs genomic integrity, and since Src and Fak are interconnected ${ }^{50}$, it will be interesting to assess whether Src inhibition triggers a similar cascade to the one described here.

Using genetic models, we reveal that Netrin-1 influences cell-fate allocation during preimplantation development (Fig. 6). Despite this effect, mouse embryos lacking zygotic Netrin-1 expression develop normally through the epiblast stage and die at E14.5 (ref. ${ }^{45}$ ). The cause of the embryonic death is currently unknown, but it coincides with the embryonic lethality in Unc5B-null mice ${ }^{23}$. It remains to be investigated whether, as with gp130, such a role may be accentuated in the context of delayed implantation ${ }^{51}$.
We show that Netrin-1 exerts different effects on MAPKs depending on the Neo1/Unc5B dosage. The repressive activity of Netrin-1 on MAPKs is converted to a stimulation by Unc5B induction, but questions remain to be answered. First, the factors triggering Unc5B induction remain to be identified. Publicly available resources indicate that transcription factors such as $\mathrm{Otx} 2$ or $\mathrm{Cdx} 2$, MyoD and Gata3 induce Unc5B when expressed in mESCs (Gene Expression Omnibus GSE31381) ${ }^{52}$. Second, the molecular mechanisms responsible for the differential effects of Netrin-1 remain to be dissected. Because these effects are mediated by the Pp2a subunit Ppp2r2c, whose paralogues Ppp2r2a and Ppp2r2d were shown to affect the Nodal pathway in opposite ways ${ }^{43}$, it will be interesting to assess whether Pp2a composition is responsible for the differential effects of Netrin-1. Finally, while Netrin-1 has been shown to mediate differential responses in various cell types ${ }^{21,22}$, our study suggests that this ability reflects a fundamental characteristic of this protein, which manifests earlier in development than previously proposed, and which might govern other cellular responses than cell migration, such as cell-fate decisions.

Collectively, our work positions Netrin-1 as a signalling pathway that participates in feedback loops with Wnt and MAPK in pluripotent cells, and demonstrates that a single ligand can trigger different effects in stem cells depending on the stoichiometry of its receptors, opening fascinating perspectives for regenerative medicine and cancer biology.

\section{Online content}

Any methods, additional references, Nature Research reporting summaries, source data, extended data, supplementary information, acknowledgements, peer review information; details of author contributions and competing interests; and statements of data and code availability are available at https://doi.org/10.1038/s41556020-0483-2.

Received: 3 November 2017; Accepted: 13 February 2020; Published online: 30 March 2020

\section{References}

1. Evans, M. J. \& Kaufman, M. H. Establishment in culture of pluripotential cells from mouse embryos. Nature 292, 154-156 (1981).

2. Martin, G. R. Isolation of a pluripotent cell line from early mouse embryos cultured in medium conditioned by teratocarcinoma stem cells. Proc. Natl Acad. Sci. USA 78, 7634-7638 (1981).

3. Dunn, S. J., Martello, G., Yordanov, B., Emmott, S. \& Smith, A. G. Defining an essential transcription factor program for naive pluripotency. Science 344, 1156-1160 (2014).

4. Williams, R. L. et al. Myeloid leukaemia inhibitory factor maintains the developmental potential of embryonic stem cells. Nature 336, 684-687 (1988).

5. Sato, N., Meijer, L., Skaltsounis, L., Greengard, P. \& Brivanlou, A. H. Maintenance of pluripotency in human and mouse embryonic stem cells 
through activation of Wnt signaling by a pharmacological GSK-3-specific inhibitor. Nat. Med. 10, 55-63 (2004)

6. Ying, Q. L., Nichols, J., Chambers, I. \& Smith, A. BMP induction of Id proteins suppresses differentiation and sustains embryonic stem cell self-renewal in collaboration with STAT3. Cell 115, 281-292 (2003).

7. Kunath, T. et al. FGF stimulation of the Erk1/2 signalling cascade triggers transition of pluripotent embryonic stem cells from self-renewal to lineage commitment. Development 134, 2895-2902 (2007).

8. Marks, H. et al. The transcriptional and epigenomic foundations of ground state pluripotency. Cell 149, 590-604 (2012).

9. Ying, Q. L. et al. The ground state of embryonic stem cell self-renewal. Nature 453, 519-523 (2008).

10. Ficz, G. et al. FGF signaling inhibition in ESCs drives rapid genome-wide demethylation to the epigenetic ground state of pluripotency. Cell Stem Cell 13, 351-359 (2013).

11. Buehr, M. et al. Capture of authentic embryonic stem cells from rat blastocysts. Cell 135, 1287-1298 (2008).

12. Li, P. et al. Germline competent embryonic stem cells derived from rat blastocysts. Cell 135, 1299-1310 (2008).

13. Choi, J. et al. Prolonged Mek1/2 suppression impairs the developmental potential of embryonic stem cells. Nature 548, 219-223 (2017).

14. Serafini, T. et al. The netrins define a family of axon outgrowth-promoting proteins homologous to C. elegans UNC-6. Cell 78, 409-424 (1994).

15. Kennedy, T. E., Serafini, T., de la Torre, J. R. \& Tessier-Lavigne, M. Netrins are diffusible chemotropic factors for commissural axons in the embryonic spinal cord. Cell 78, 425-435 (1994).

16. Cirulli, V. \& Yebra, M. Netrins: beyond the brain. Nat. Rev. Mol. Cell Biol. 8, 296-306 (2007).

17. Grandin, M. et al. Structural decoding of the netrin-1/UNC5 interaction and its therapeutical implications in cancers. Cancer Cell 29, 173-185 (2016).

18. Bell, C. H. et al. Structure of the repulsive guidance molecule (RGM)neogenin signaling hub. Science 341, 77-80 (2013).

19. Rajagopalan, S. et al. Neogenin mediates the action of repulsive guidance molecule. Nat. Cell Biol. 6, 756-762 (2004).

20. $\mathrm{Xu}, \mathrm{K}$. et al. Neural migration. Structures of netrin-1 bound to two receptors provide insight into its axon guidance mechanism. Science 344, 1275-1279 (2014).

21. Ko, S. Y., Dass, C. R. \& Nurgali, K. Netrin-1 in the developing enteric nervous system and colorectal cancer. Trends Mol. Med. 18, 544-554 (2012)

22. Hong, K. et al. A ligand-gated association between cytoplasmic domains of UNC5 and DCC family receptors converts netrin-induced growth cone attraction to repulsion. Cell 97, 927-941 (1999).

23. Lu, X. et al. The netrin receptor UNC5B mediates guidance events controlling morphogenesis of the vascular system. Nature 432, 179-186 (2004).

24. Ozmadenci, D. et al. Netrin-1 regulates somatic cell reprogramming and pluripotency maintenance. Nat. Commun. 6, 7398 (2015).

25. Rajasekharan, S. \& Kennedy, T. E. The netrin protein family. Genome Biol 10, 239 (2009).

26. Wray, J. et al. Inhibition of glycogen synthase kinase-3 alleviates Tcf3 repression of the pluripotency network and increases embryonic stem cell resistance to differentiation. Nat. Cell Biol. 13, 838-845 (2011).

27. Skarnes, W. C., Moss, J. E., Hurtley, S. M. \& Beddington, R. S. Capturing genes encoding membrane and secreted proteins important for mouse development. Proc. Natl Acad. Sci. USA 92, 6592-6596 (1995).

28. Kumar, R. M. et al. Deconstructing transcriptional heterogeneity in pluripotent stem cells. Nature 516, 56-61 (2014).

29. Guo, G. et al. Serum-based culture conditions provoke gene expression variability in mouse embryonic stem cells as revealed by single-cell analysis. Cell Rep. 14, 956-965 (2016).
30. Bulut-Karslioglu, A. et al. Inhibition of mTOR induces a paused pluripotent state. Nature 540, 119-123 (2016).

31. Boroviak, T. et al. Lineage-specific profiling delineates the emergence and progression of naive pluripotency in mammalian embryogenesis. Developmental Cell 35, 366-382 (2015).

32. Galonska, C., Ziller, M. J., Karnik, R. \& Meissner, A. Ground state conditions induce rapid reorganization of core pluripotency factor binding before global epigenetic reprogramming. Cell Stem Cell 17, 462-470 (2015).

33. Habibi, E. et al. Whole-genome bisulfite sequencing of two distinct interconvertible DNA methylomes of mouse embryonic stem cells. Cell Stem Cell 13, 360-369 (2013).

34. von Meyenn, F. et al. Impairment of DNA methylation maintenance is the main cause of global demethylation in naive embryonic stem cells. Molecular Cell 62, 848-861 (2016).

35. Beurel, E., Grieco, S. F. \& Jope, R. S. Glycogen synthase kinase-3 (GSK3): regulation, actions, and diseases. Pharmacol. Ther. 148, 114-131 (2015).

36. Guenebeaud, C. et al. The dependence receptor UNC5H2/B triggers apoptosis via PP2A-mediated dephosphorylation of DAP kinase. Molecular Cell 40, 863-876 (2010)

37. Ren, X. R. et al. Focal adhesion kinase in netrin-1 signaling. Nat. Neurosci. 7, 1204-1212 (2004).

38. Liu, G. et al. Netrin requires focal adhesion kinase and Src family kinases for axon outgrowth and attraction. Nat. Neurosci. 7, 1222-1232 (2004).

39. Moore, S. W. \& Kennedy, T. E. Protein kinase A regulates the sensitivity of spinal commissural axon turning to netrin-1 but does not switch between chemoattraction and chemorepulsion. J. Neurosci. 26, 2419-2423 (2006).

40. Qu, C. et al. c-Jun N-terminal kinase 1 (JNK1) is required for coordination of netrin signaling in axon guidance. J. Biol. Chem. 288, 1883-1895 (2013).

41. Gao, C. et al. FAK/PYK2 promotes the Wnt/ $\beta$-catenin pathway and intestinal tumorigenesis by phosphorylating GSK3 $\beta$. eLife 4, e10072 (2015).

42. Sangodkar, J. et al. All roads lead to PP2A: exploiting the therapeutic potential of this phosphatase. FEBS J. 283, 1004-1024 (2016).

43. Batut, J. et al. Two highly related regulatory subunits of PP2A exert opposite effects on TGF- $\beta$ /Activin/Nodal signalling. Development 135, 2927-2937 (2008).

44. Dominici, C. et al. Floor-plate-derived netrin-1 is dispensable for commissural axon guidance. Nature 545, 350-354 (2017).

45. Bin, J. M. et al. Complete loss of netrin-1 results in embryonic lethality and severe axon guidance defects without increased neural cell death. Cell Rep. 12, 1099-1106 (2015).

46. Nakamura, T. et al. A developmental coordinate of pluripotency among mice, monkeys and humans. Nature 537, 57-62 (2016).

47. Heffner, C. S. et al. Supporting conditional mouse mutagenesis with a comprehensive cre characterization resource. Nat. Commun. 3, 1218 (2012).

48. Hayashi, K., Ohta, H., Kurimoto, K., Aramaki, S. \& Saitou, M. Reconstitution of the mouse germ cell specification pathway in culture by pluripotent stem cells. Cell 146, 519-532 (2011)

49. ten Berge, D. et al. Embryonic stem cells require Wnt proteins to prevent differentiation to epiblast stem cells. Nat. Cell Biol. 13, 1070-1075 (2011)

50. Mitra, S. K. \& Schlaepfer, D. D. Integrin-regulated FAK-Src signaling in normal and cancer cells. Curr. Opin. Cell Biol. 18, 516-523 (2006).

51. Nichols, J., Chambers, I., Taga, T. \& Smith, A. Physiological rationale for responsiveness of mouse embryonic stem cells to gp130 cytokines. Development 128, 2333-2339 (2001).

52. Correa-Cerro, L. S. et al. Generation of mouse ES cell lines engineered for the forced induction of transcription factors. Sci. Rep. 1, 167 (2011).

Publisher's note Springer Nature remains neutral with regard to jurisdictional claims in published maps and institutional affiliations.

(c) The Author(s), under exclusive licence to Springer Nature Limited 2020 


\section{Methods}

Animal studies. Teratoma assays were performed with 7-week-old severe combined immunodeficient (SCID) male mice (CB17/SCID, Charles River). $N t n 1^{\beta g e o}$ reporter and Netrin-1 conditional-KO mESCs were derived from C57/bl6 mixed-background pregnant females at 8-15 weeks of age. Blastocyst injections were done using BALB/cANRj embryos. Embryos were flushed with M2 medium (Sigma) and grown overnight in KSOM (Sigma) or sequential blast (Origio) medium. Genotyping of $N t n 1^{f / f l}$ embryos was performed as previously described ${ }^{44}$. $\mathrm{X}$-gal was detected in blastocysts using secondary antibodies coupled with biotin, the Vectastain ABC kit and DAB (Vector System). Teratoma-formation assays were performed by injecting $1 \times 10^{6} \mathrm{mESCs}$ in the testes of 7 -week-old SCID mice. After 3-4 weeks, the mice were euthanized and lesions were surgically removed and fixed in formalin or in $4 \%$ paraformaldehyde for sections. For blastocyst injections, $N \operatorname{tn} 1^{f l / f l}$, untreated or treated with TAM for $48 \mathrm{~h}$, were injected into BALB/cANRj blastocysts. The day before injection, frozen BALB/cANRj morulas from Quickblasto (Janvier) were thawed according to the manufacturer's instructions and incubated overnight in KSOM medium (Millipore) at $37^{\circ} \mathrm{C}$ with $5 \% \mathrm{CO}_{2}$. Between 5 and 15 cells were injected into expanded blastocysts in M2 medium (Sigma) using standard blastocyst injection techniques. Blastocysts were then allowed to recover for a period of $1-3 \mathrm{~h}$ prior to being implanted into pseudopregnant females. All animal procedures were performed in accordance with institutional guidelines (French ceccapp project 01369.01).

Cell culture. The following cell lines were used in the study. Cgr8 ES cells (ECACC 07032901) were provided by the B. Pain laboratory (SBRI, Bron, France). E14Tg2a ES cells (ATCC CRL 1821) were provided by the M. E. Torres Padilla laboratory (IES, Munchen, Germany). Ntn $1^{\text {Bgeo }}$ reporter $^{27}$ and Netrin-1 conditional-KO $\left(N \operatorname{tn} 1^{f l / f}\right)^{44}$ mESCs were derived from C57/bl6 mixed-background mice for the study, with no further authentication. Netrin-1 depletion in Ntn $1^{f l f l}$ mESCs was induced by treatment with $0.2 \mu \mathrm{M} 4$ '-OH-tamoxifen (TAM) for $48 \mathrm{~h}$. Cgr8 and E14Tg2a ES cells were cultured on gelatin as previously described ${ }^{24}$. Control, Netrin1(WT), Netrin-1(Unc5B-mut) and Netrin-1(Neol-mut) were established from the same starting Cgr8 population. The Netrin-1(KO), Neol(KO), Unc5B(KO) and Netrin-1-doxi mESCs were generated by stable transfection of Cgr8 mESCs using FugeneHD reagent (Promega) or Lipofectamine 2000 (Life technologies). Netrin-1 revertant mESCs were obtained by treating cells with 100 units TAT-Cre (SCR-508, Millipore) for $24 \mathrm{~h}$ followed by FACS of GFP-expressing cells. Control Neo1-doxi, Netrin-1 Neo1-doxi, control Unc5B-doxi and Netrin-1 Unc5B-doxi mESCs were generated by infecting control and Netrin-1-expressing mESCs with doxi plasmids (a kind gift from the Mehlen laboratory). The siRNA (Dharmacon) were reverse transfected in Cgr8 ES cells at a final concentration of $30 \mathrm{nM}$ using lipofectamine 2000. Silenced Negative Control siRNA (Life Technologies) was used as negative control for siRNA transfection. Cells were collected 2-3 d after transfection. siRNA sequences are detailed in Supplementary Table 1. The hiPS cells were generated from human dermal fibroblasts (Merck 106-05A) using Sendai viruses, cultured in complete TeSR-E8 medium on vitronectin-coated plates (StemCell Technologies). Medium was changed daily and cells were passaged once a week in the presence of $10 \mu \mathrm{M}$ ROCK inhibitor Y-27632 (Sigma-Aldrich) with Ultra-Pure EDTA solution (Invitrogen). All cell lines used in the study were tested mycoplasma-free (using the Mycoalert kit). The following recombinant proteins were used as follows: mouse Wnt3a (R\&D Systems, 1324-WN) 50 ng ml $^{-1}$, human Fgf4 (Peprotech, 100-31) $10 \mathrm{ng} \mathrm{ml}^{-1}$ and human Activin (Invitrogen, PHG9014). The $293 \mathrm{~T}$ and plat-E cells were grown in DMEM supplemented with $10 \%$ FCS and penicillin-streptomycin. PD0325901 (Millipore, 444968) and CHIR99021 (Millipore, 361571) were purchased from Merck Millipore. Luciferase assays were performed using dual luciferase reporter assay system (Promega E1910). Wild-type Netrin-1 from Gallus gallus, lacking the C-terminal domain (NP_990750, amino acid residues 26-458) was cloned into a modified PCEP vector with an C-terminal Strep-II tag. HEK293 cells were stably transfected and secreted Netrin-1 was purified by streptavidin chromatography (IBA) followed by the removal of the tag by thrombin digestion. Purified Netrin-1 was then dialysed against PBS and passed through a sterile filter. Protein concentration was corrected using the calculated extinction coefficients for Netrin-1 (ProtParam utility available on the ExPaSy server).

Self-renewal and exit from pluripotency assays. For colony-formation assay, mESCs were plated at clonal density (60-100 cells per $\mathrm{cm}^{2}$, depending on the strain) in serum/Lif on gelatin-coated plates. Medium was changed every day for $7 \mathrm{~d}$ before detection of alkaline phosphatase-positive colonies (AP0100-1KT, Sigma). For exit-from-pluripotency assays, mESCs were grown for $7 \mathrm{~d}$ in serum without Lif on gelatin, then split and replated for $7 \mathrm{~d}$ more in serum/Lif. For selfrenewal assays, E14Tg2a mESCs were plated at clonal density on laminin-coated dishes and split every $3 \mathrm{~d}$.

Differentiation assays. Serum deprivation and stimulation experiments were conducted by growing mESCs overnight in N2B27 medium without cytokines, followed by exposure to the appropriate molecules for the times indicated in the figures. Control Neo1-doxi and Netrin-1 Neo1-doxi mESCs were grown in N2B27 + doxycycline medium for $24 \mathrm{~h}$ prior to collection and embryoid body-formation assays were carried out by growing mESCs in non-adherent culture conditions in non-treated plastic plates for the indicated times. EpiLC induction was performed as previously described ${ }^{18}$.

Constructs. To perform in situ hybridization on mouse embryos, a Ntn1 probe was cloned from mESC cDNA into pGEMTeasy (Promega) (sequence available on request) and in situ hybridization was performed as previously described. Point mutations were introduced into the pcagg-Netrin-1-ires-puro vector to generate mutant Netrin-1 versions using the quick site-directed mutagenesis kit (Agilent). CRISPR-KO plasmids were engineered using the backbone pSpCas9 (BB)-2A-Puro and the protocol from the Zhang lab (Ran et al., 2013). The small guide RNAs were designed using the UCSC genome browser and CRISPOR websites. Guides are detailed in Supplementary Table 1. Neo1- and Unc5B-doxi pITR vectors were kind gifts from P. Mehlen laboratory (CRCL, Lyon, France).

Antibodies, RT-qPCR and biochemical assays. Primary antibodies used in this study are detailed in Supplementary Table 1. The main antibodies were validated using gain- and loss-of-function approaches. Membrane fractionation was performed by using Mem-PER Plus Membrane Protein Extraction Kit (Thermofisher 89842). Nuclear and cytoplasmic extraction was performed with the NE-PER Nuclear and Cytoplasmic Extraction Reagents Kit (Thermofisher 78833). Pp2a activity was measured using an immunoprecipitation-based method (Millipore 17-313). RT-qPCR, immunofluorescence and western blots were performed as previously described ${ }^{24}$. Primers are presented in Supplementary Table 2.

Deep sequencing. For RNA-sequencing (RNA-seq) experiments, RNA quality was analysed using a Bioanalyser (Agilent). Libraries were constructed and sequenced on an illumina Hiseq 2000 (Beckman Coulter Genomics and Genewiz). ChIP-seq experiments were performed as previously described ${ }^{32}$. For ChIP-seq analysis, BWA was used for alignment of data to the $\mathrm{mm} 9$ genome with de-duplication performed using Picard tools, followed by peak calling using macs 2 with narrow peak settings. To compare between control and Netrin-1 conditions, Homer was used to create tag directories from aligned reads, then annotatePeaks.pl was used with size $=8,000$ and hist $=10$ for all plots except $\mathrm{H} 3 \mathrm{~K} 27 \mathrm{me} 3$ global enrichment, where size $=50,000$ and hist $=200$ was used due to the wider distribution. Bed files used for these analyses included bivalent promoters defined in ref. ${ }^{32}$ and ENCODEdefined $\mathrm{H} 3 \mathrm{~K} 4 \mathrm{me} 3$ and $\mathrm{H} 3 \mathrm{~K} 27 \mathrm{me} 3$ peaks in Bruce 4 ES cells (ENCFF001XWU and ENCFF001XWQ). Data were then plotted using R. IGV was used for visualization of ChIP-seq enrichment (expressed in fragments per kilobase of transcript per million (FKPM)). For analysis of restricted representation bisulfite-sequencing data, UCSC liftOver was used to convert coordinates from $\mathrm{mm} 10$ to $\mathrm{mm} 9$ and the two replicates for control and Netrin-1 samples were averaged at matching CpGs. For comparison to $2 \mathrm{i}$ conditions, WGBS data from Gene Expression Omnibus dataset GSM1027572 were used. Control, Netrin-1 and $2 \mathrm{i}$ data were then merged so that only matched CpGs with coverage of at least $5 \times$ (for control and Netrin-1) and $1 \times$ (for $2 \mathrm{i}$ ) were used. Violin plots were generated in R using the library 'Vioplot' v.0.2 and heat maps were made using custom scripts in R. BedTools v.2.25.0 was used to intersect CpGs with the following genomic features: high CpG promoters, CpG islands, low $\mathrm{CpG}$ promoters, $\mathrm{CpG}$ island shores, exons, introns, long interspersed nuclear elements, short interspersed nuclear elements, long terminal repeats and intracisternal A-particle elements, with all annotation downloaded from UCSC with the exception of $\mathrm{CpG}$ islands, low $\mathrm{CpG}$ promoters and high $\mathrm{CpG}$ promoters, which were computationally assigned. For any CpGs located within these features, mean methylation was calculated and plotted in $\mathrm{R}$.

Hierarchical clustering. Control and Netrin-1 mESC datasets were processed as follows. The primary assembly Mus musculus genome sequence (release GRCm38.p5) and transcriptome annotations (Ensembl release 87) were downloaded from the GENCODE website. Raw read data (fastq files) were mapped to these sequences using STAR (with parameters -outFilterType BySJout, -outSAMtype BAM Unsorted, and -quantMode GeneCounts). This last parameter enables direct conversion of the mappings to gene counts. These gene counts were transformed into FPKM and combined with the table provided ${ }^{7}$. Only gene names present in both datasets were kept. The following process was applied: (1) keep genes with an average FPKM of at least 10 in at least one cell type (resulting in, as reported, 9,639 genes); (2) normalize between datasets by subtracting, for each gene, the average $\log _{2}(F P K M+1)$ in each dataset from this gene and $\log _{2}(F P K M+1)$ in the corresponding samples (geometric mean); (3) compute a dissimilarity matrix between samples by using the 1 - Spearman correlation between samples; and (4) generate a hierarchical clustering using the 'average' agglomeration method.

FACS. FACS analysis was performed on a BD LSRFortessa. Sorting was performed on a BD FACSDiVa. Cells were sorted, washed immediately and centrifuged before being plated directly in fresh medium or frozen for RNA extraction and gene expression analysis.

Statistics and reproducibility. Western blot quantifications were performed with ImageJ. Statistical analyses of mean and variance were performed with Prism 6 (GraphPad Software) and Student's $t$-test or Wilcoxon tests where indicated. For western blots presented in the figures, three independent experiments gave similar results. 
Reporting Summary. Further information on research design is available in the Nature Research Reporting Summary linked to this article.

\section{Data availability}

Deep sequencing and ChIP-seq data that support the findings of this study have been deposited in the Gene Expression Omnnibus under accession code GSE102831. Previously published sequencing data that were re-analysed here are available under accession code E-MTAB-2958, E-MTAB-2959 (ref. ${ }^{31}$ ), GSE81285 (ref. ${ }^{30}$ ) and GSE31381 (ref. ${ }^{52}$ ). All other data supporting the findings of this study are available from the corresponding author on reasonable request.

\section{Acknowledgements}

We are grateful to the PBES Lyon for technical assistance. We thank V. Azuara and D. Stupack for critical reading of the manuscript and L. Favre-Louis for technical assistance. This work was supported by institutional grants from INSERM/CNRS, Atip-avenir, Plan cancer, La ligue contre le cancer nationale et régionale (F.L.), INCa (F.L.), Fondation ARC (F.L., G.F. and D.O.), Centre Léon Bérard (F.L. and A.H.), Fondation pour la recherche médicale (F.L.), National Institutes of Health (R01-HD081534 (B.J.M.)), ANR (P.M.), ERC (P.M.) Max Planck Society (A.M.) and the DFG Forschergruppe 2722 (M.K.).

\section{Author contributions}

A.H. and G.F. performed most of the experiments in Figs. 1-7. D.O. performed experiments in Figs. 1, 5 and 7. X.G. and N.C. performed experiments in Figs. 1, 3 and 5. A.M., C.G. and C.R. performed and analysed ChIP-seq experiments. J.C. and N.R. carried out the bioinformatic analyses. M.K. and T.I. produced recombinant Netrin-1 used in Figs. 4 and 5. P.W. performed teratoma experiments. F.L., A.H., G.F. and D.O. designed experiments. F.L. initiated, designed and supervised the study. F.L. wrote the manuscript.

\section{Competing interests}

The authors declare no competing interests.

\section{Additional information}

Extended data is available for this paper at https://doi.org/10.1038/s41556-020-0483-2. Supplementary information is available for this paper at https://doi.org/10.1038/ s41556-020-0483-2.

Correspondence and requests for materials should be addressed to F.L.

Reprints and permissions information is available at www.nature.com/reprints. 
a
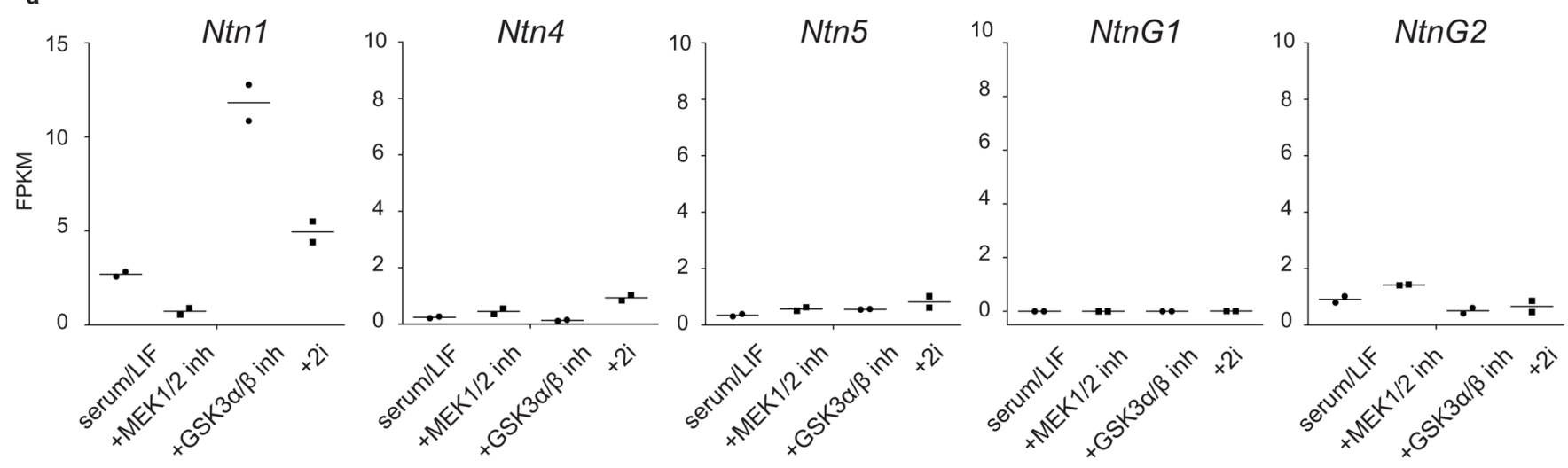

b

c

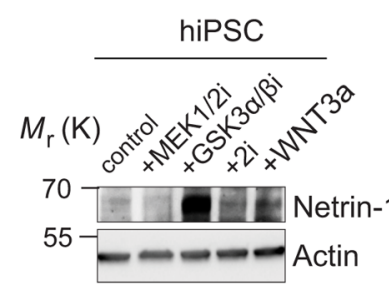

Ntn1

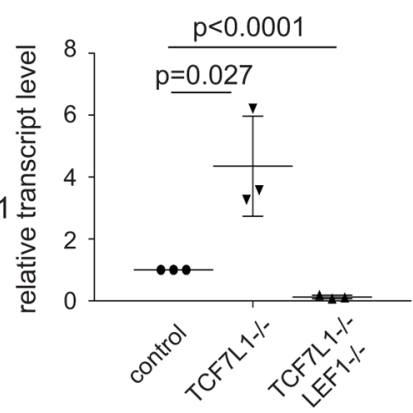

d

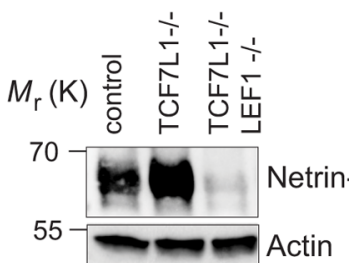

e

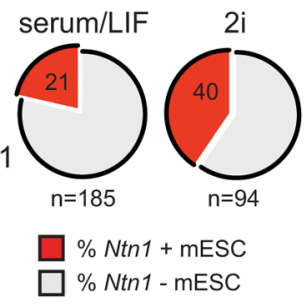

Ntn1

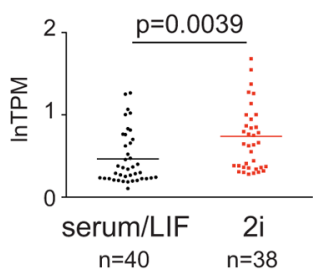

Extended Data Fig. 1 | Netrin-1 is expressed in naive pluripotent cells in vitro. (a) Data present FPKM values for Ntn1, Ntn4, Ntn5, NtnG1 and NtnG2 in serum/Lif mESCs treated or not with Mek1/2-inh (PD), Gsk3 $\alpha / \beta$-inh (CHIR) or both (2i). 2 independent experiments. (b) Western blot depicting netrin-1 levels in human iPS cells treated similarly as (a) (3 independent experiments). (c) Netrin-1 transcripts level in indicated mESCs. Q-RTPCR data are expressed relative to $\mathrm{mESC}$ as the mean \pm s.d. ( $n=3$ independent experiments). Student's t-test was used and two-tailed $p$-values are indicated. (d) Netrin-1 western blot in indicated mESCs (3 independent experiments). (e) Netrin-1 expression in single mESCs in Serum/Lif and 2i. Single-cell transcriptomic data are extracted from Kumar et al., 2014, ref. ${ }^{28} \cdot \mathrm{n}=$ number of cells analysed in each condition. (f) Netrin-1 mean expression in single mESCs. Data are extracted from Kumar et al., 2014 , ref. ${ }^{28} . n=$ number of cells analysed in each condition. The bar represents the mean $\pm \mathrm{s} . \mathrm{d}$. of netrin-1 expression in the 2 conditions. Student t-test was used and two-sided p-value is indicated. 
a

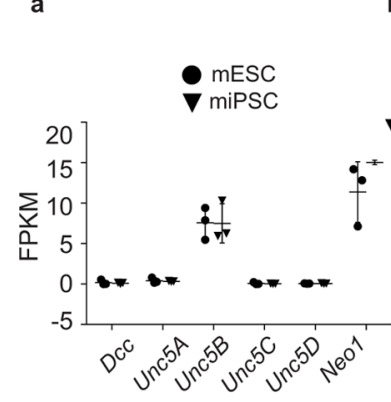

e
E2.5 morula

E3.5 ICM

E4.5 epiblast

E5.5 epiblast d

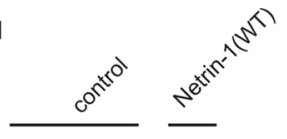

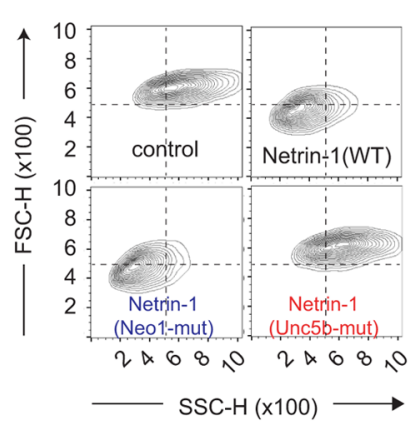

j g

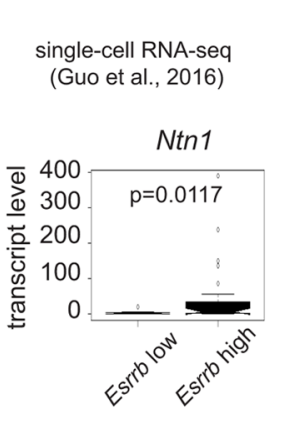

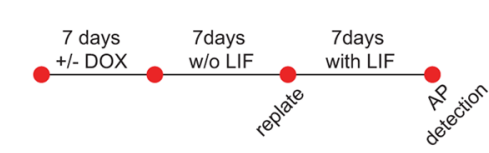

h

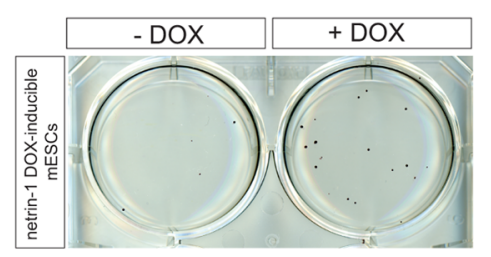

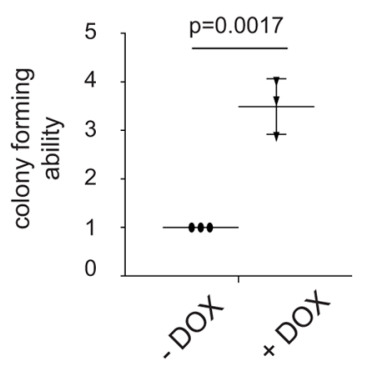
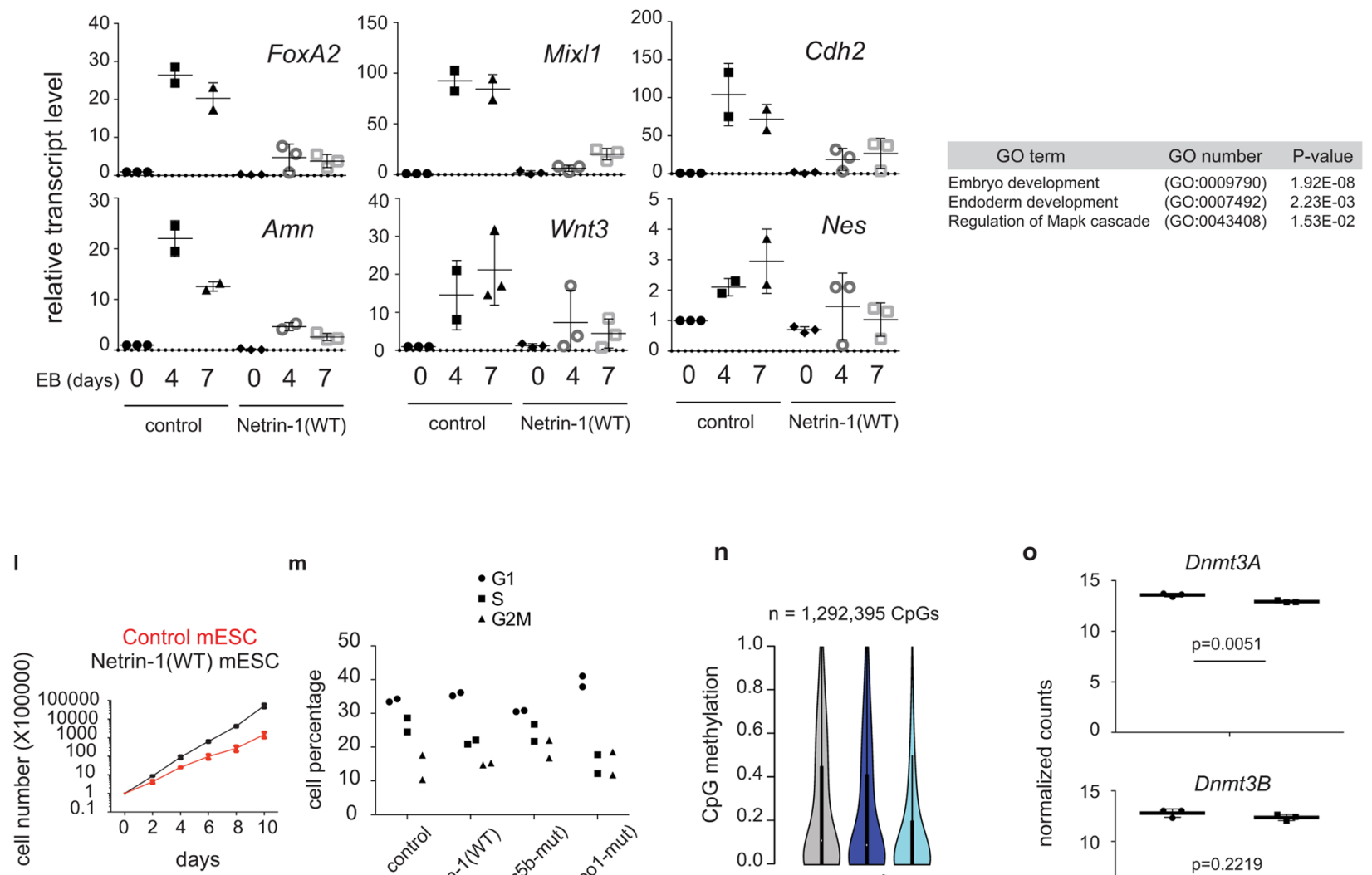

m

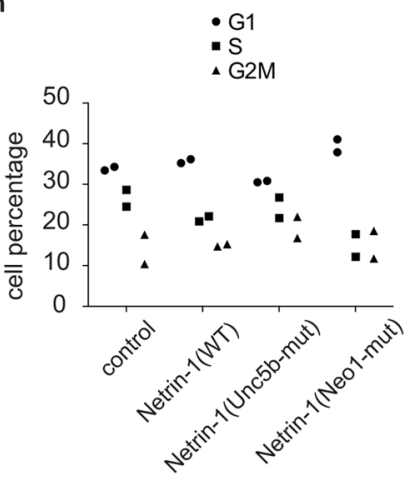

n

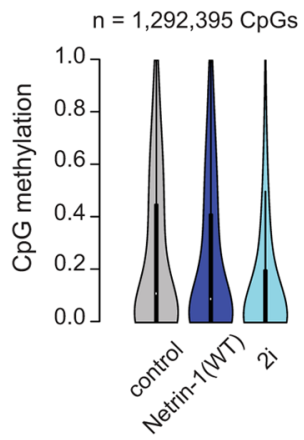

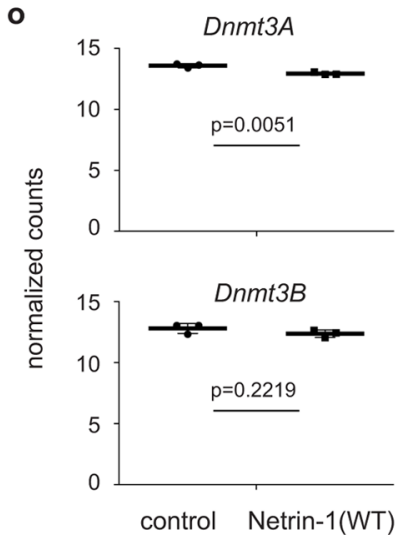

Extended Data Fig. 2 | See next page for caption. 
Extended Data Fig. 2 | Netrin-1 triggers pluripotency features partially overlapping with the ground state. (a) Netrin-1 receptors transcript levels in mouse ES and iPS cells. RNA-seq data are presented as FPKM values and expressed as the mean \pm s.d. ( $n=3$ independent experiments). (b) Netrin-1 receptors expression during early mouse development. Data, extracted from Boroviak. et al., 2015, ref. ${ }^{31}$, present transcripts level in FPKM. Data are presented as the mean \pm s.d. ( $n=3$ independent experiments). (c) Western blot performed in the indicated cell lines ( 3 independent experiments). (d) Western blot performed in the indicated mESCs grown in serum/Lif (3 independent experiments). (e) FACS analysis (FSC/SSC) of the different populations grown in serum/Lif. (f) netrin-1 expression in ES cells subpopulations. Data are extracted from Guo et al. 2016, ref. ${ }^{29}$. Esrrb expression is used to distinguish quartiles of Esrrb ${ }^{\text {high }}(>Q 1)$ and Esrrb ${ }^{\text {low }}(<Q 1)$ cells. netrin-1 expression is analysed in the corresponding quartile. $n=48$ total cells were analysed, 12 cells for $<Q 1$ and 36 cells for $>Q 1$. Student's t-test was used and two-tailed p-value is indicated. (g) Scheme depicting exit from pluripotency assays. (h) Pictures of a single experiment representative of three independent ones. (i) Colony counting. Data are the mean $\pm s . d$. ( $n=3$ independent experiments). Student's t-test was used and two-sided p-values are indicated. (j) Q-RTPCR depicts transcript level in mESCs and EBs generated with indicated cell lines. Data are the mean \pm s.d. $n=3$ independent experiments with exclusion of outliers. (k) Statistical overrepresentation analysis. Panther DB was used to detect overrepresented GO within differentially expressed genes. A Fisher's exact two-sided test was used to calculate $p$-values. $n=3$ independent samples. (I) Proliferation curves. Data are the mean \pm s.d. of 2 independent experiments. ( $\mathbf{m}$ ) Cell cycle features. Data are the mean \pm s.d. ( $n=2$ independent experiments). (n) Violin plots displaying methylation levels for $n=1.3 \mathrm{M}$ matched CpGs. Bold line indicates $25-75$ th percentile, white dot indicates median. (o) Dnmt3A and Dnmt3B expression levels. Data are the mean \pm s.d. ( $n=3$ independent experiments) of normalized counts. Student's T test was used and two-sided p-values are indicated. 


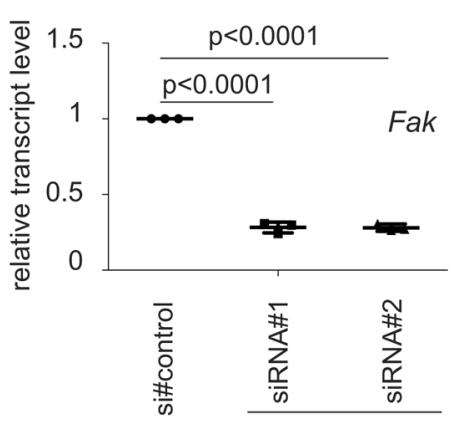

Fak

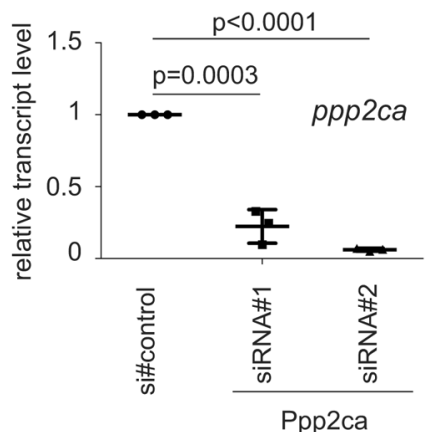

Extended Data Fig. 3 | Molecular cascade downstream of Netrin-1 in mESCs. (a) Knockdown efficiency of Fak in mESCs. Q-RTPCR depicts Fak transcript level following transfection of netrin-1 mESCs with independent siRNA. Data, normalised to si\#control mESCs, are the mean $+/-$ sd of $n=3$ independent experiments. Student T-test was used and two-sided p-values are indicated. (b) Effect of netrin-1 signalling on Lif sensitivity. Control and netrin-1 (WT) mESCs were serum-starved ON and stimulated with Lif for 10 mins prior to samples collection (3 independent experiments). (c) Knockdown efficiency of Ppp2ca and Ppp2r2c in mESCs. Similar settings as (a). Data, normalised to si\#control mESCs, are the mean $+/-$ sd of $n=3$ independent experiments. Student T-test was used and two-sided $p$-values are indicated. 
a

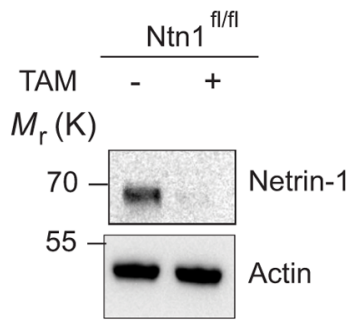

b

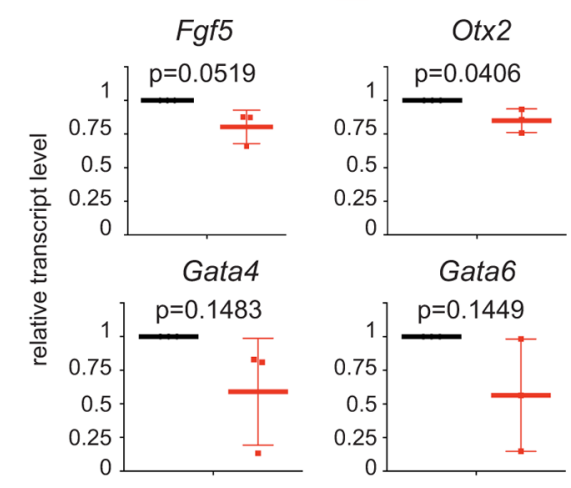

c

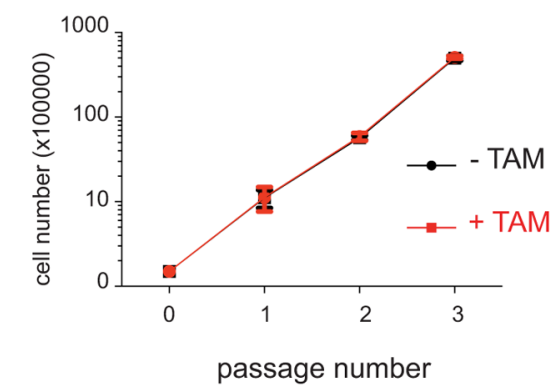

e

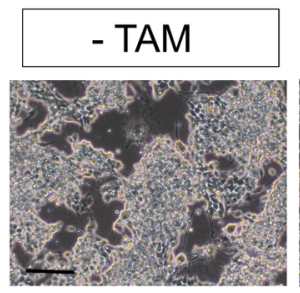

+ TAM

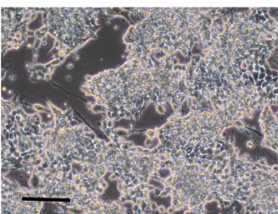

PI

f

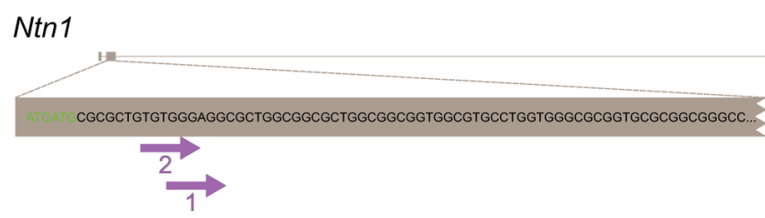

Unc5b

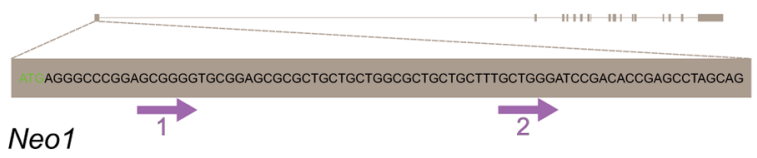

g

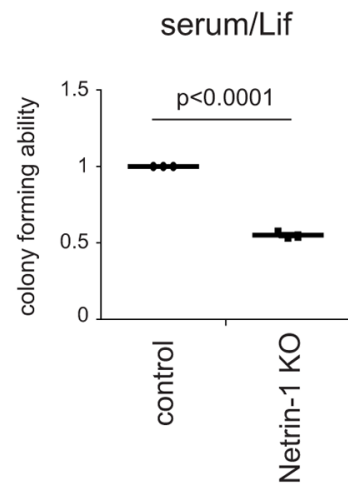

serum/Lif+2i

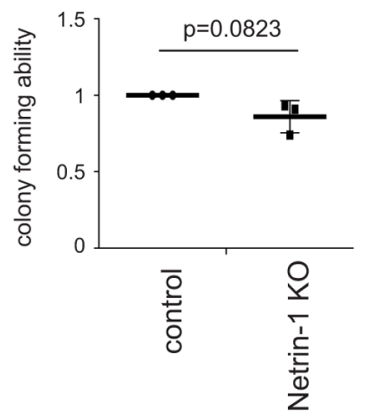

h

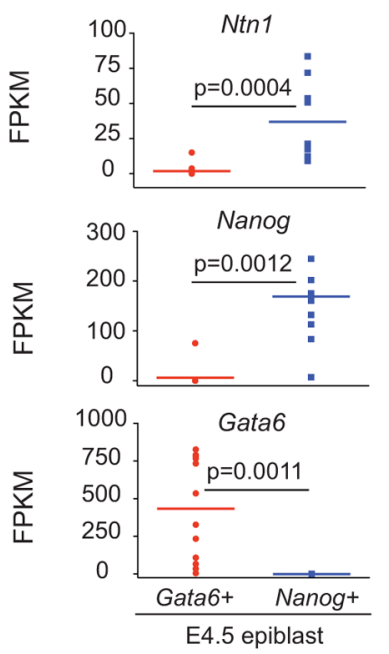

Extended Data Fig. 4 | See next page for caption. 
Extended Data Fig. 4 | Endogenous Netrin-1 controls pluripotency features. (a) Western blot in Ntn ${ }^{1 / 1 / f l} \mathrm{mESC}$ treated or not with 4'OH-tamoxifen (TAM) for 3 days before collection (3 independent experiments). (b) Q-RTPCR depicts Fgf5, Otx2, Gata4 and Gata6 transcript level following netrin-1 depletion in $\mathrm{mESC}$. Data are the mean $+/-\mathrm{sd}$ of $\mathrm{n}=3$ independent experiments. Student T-test was used and two-sided $p$-values are indicated. (c) Proliferation curves. The Ntn $1^{f / / f l} \mathrm{mESC}$, treated or not with TAM, were counted at each passage in serum/Lif. Data are the mean $+/-\mathrm{sd}$ of $\mathrm{n}=3$ independent experiments. (d) Cell death analysis. The Ntn1 $1^{\mathrm{fl} / \mathrm{fl}} \mathrm{mESC}$, treated or not with TAM, were grown for 2 days in N2B27+Lif before PI-AnnexinV staining was performed. The left panel presents a representative FACS profile and the right panel a graph of mean data $\pm s . d$. ( $n=3$ independent experiments). Value $100 \%$ is given to the percentage of live cells in untreated Ntn $1^{\mathrm{fl} / \mathrm{fl}} \mathrm{mESCs}$. Student T-test was used and two-sided $\mathrm{p}$-values are indicated. (e) Brightfield pictures of $\mathrm{Ntn} 1^{\mathrm{fl} / \mathrm{fl}} \mathrm{mESCs}$ treated or not with $4^{\prime} \mathrm{OH}$-tamoxifen and subsequently maintained in culture for 22 passages. Bars: $50 \mu \mathrm{m} .3$ independent experiments. (f) Scheme of the crispr/cas9 guides. The grey boxes correspond to exons, and pink arrows indicate the 2 independent guides for each locus. (g) Self-renewal assay. Control and netrin-1 KO mESCs are plated at clonal density in serum+Lif (left panel) or serum+Lif+2i (right panel) for 7 days before AP positive colonies was scored. Data are mean \pm s.d. ( $n=3$ independent experiments). Student's t-test was used and two-sided p-values are indicated. (h) Gene expression in single blastomeres. Data, extracted from Nakamura et al., 2016 (ref. ${ }^{46}$ ), correspond to FPKM values. $n=9$ nanog positive cells $n=12$ gata 6 positive blastomeres. Each dot corresponds to a cell, the bar is the mean \pm s.d. Student T-test was used and two-sided p-values are indicated. 
a

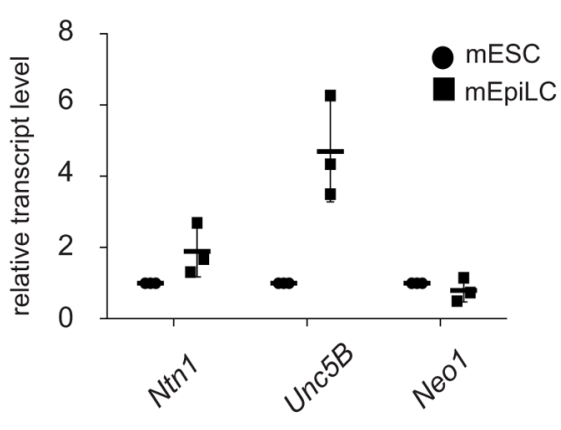

d
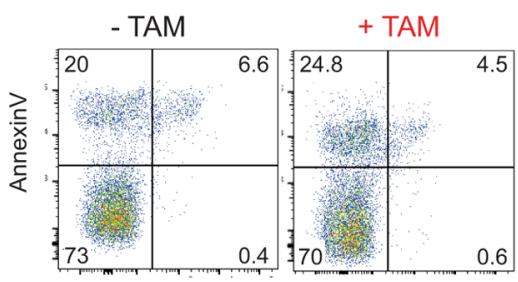

$\mathrm{Pl}$ b

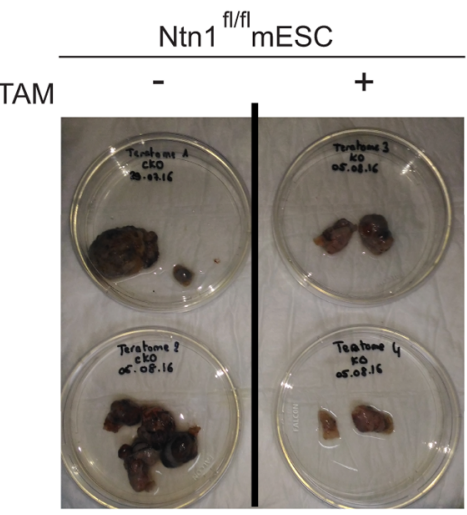

- TAM

+ TAM

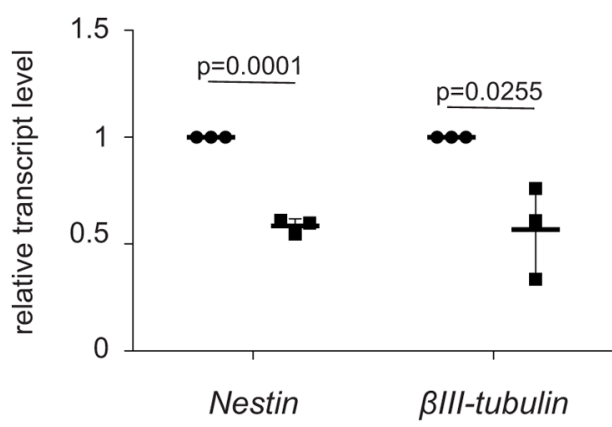

Extended Data Fig. 5 | Netrin-1 controls coordinated differentiation. (a) Neo1 and Unc5B expression in epiblast-like cells (EpiLC). Q-RTPCR data are expressed relative to $\mathrm{mESC}$ s as the mean \pm s.d. ( $n=3$ independent experiments). (b) Pictures of teratoma obtained following injection of Ntn $1^{f / 1 / 1 /} \mathrm{mESCs}$ treated (right panel) or not (left panel) with TAM 24 hours prior to injection. 4 independent teratoma per condition were analysed. (c) Q-RTPCR depicts Nestin and $\beta$ III-tubulin levels at day 8 of differentiation in N2B27-Lif. Data are normalized to housekeeping genes and value 1 is given to day8 Ctrl mESCs. Data are the mean \pm s.d. ( $n=3$ independent experiments). Student's t-test was used and two-sided $p$-values are indicated. (d) Cell death analysis. The Ntn $1^{f / f f l}$ mESCs, treated or not with TAM, were grown for 2 days in N2B27-Lif before PI-AnnexinV staining was performed. The left panel present a representative FACS profile and the right panel a graph of mean data \pm s.d. ( $n=3$ independent experiments). Value $100 \%$ is given to the percentage of live cells in untreated $\mathrm{Ntn} 1^{f / 1 / 1}$ mESCs. Student T-test was used and two-sided $\mathrm{p}$-values are indicated. 


\section{Reporting Summary}

Nature Research wishes to improve the reproducibility of the work that we publish. This form provides structure for consistency and transparency in reporting. For further information on Nature Research policies, see Authors \& Referees and the Editorial Policy Checklist.

\section{Statistical parameters}

When statistical analyses are reported, confirm that the following items are present in the relevant location (e.g. figure legend, table legend, main text, or Methods section).

$\mathrm{n} / \mathrm{a} \mid$ Confirmed

$\square \bigotimes$ The exact sample size $(n)$ for each experimental group/condition, given as a discrete number and unit of measurement

$\square$ An indication of whether measurements were taken from distinct samples or whether the same sample was measured repeatedly

$\square$ The statistical test(s) used AND whether they are one- or two-sided

Only common tests should be described solely by name; describe more complex techniques in the Methods section.

Х A description of all covariates tested

$\square$ \A description of any assumptions or corrections, such as tests of normality and adjustment for multiple comparisons

$\square$ A full description of the statistics including central tendency (e.g. means) or other basic estimates (e.g. regression coefficient) AND

$\triangle$ variation (e.g. standard deviation) or associated estimates of uncertainty (e.g. confidence intervals)

$\square$ For null hypothesis testing, the test statistic (e.g. $F, t, r$ ) with confidence intervals, effect sizes, degrees of freedom and $P$ value noted

Give P values as exact values whenever suitable.

Х $\square$ For Bayesian analysis, information on the choice of priors and Markov chain Monte Carlo settings

$\square$ For hierarchical and complex designs, identification of the appropriate level for tests and full reporting of outcomes

Х Estimates of effect sizes (e.g. Cohen's $d$, Pearson's $r$ ), indicating how they were calculated

$\varnothing$ Clearly defined error bars

State explicitly what error bars represent (e.g. SD, SE, CI)

Our web collection on statistics for biologists may be useful.

\section{Software and code}

Policy information about availability of computer code

Data collection Western blot acquisition was performed using a Image Lab Bio-Rad software. Q-RTPCR data were acquired using the Lightcycler 4.1 software. Microscopy images were acquired with axiovision 4.8.2 software. FACS data were acquired on a FACSDiva v8.0 software.

Data analysis

Quantification of western blot data was performed using ImageJ. Most of the data analysis and statistics were performed using Graphpad prims 6. ChIP-seq data were analysed using BWA, Picard, macs2, custom R scripts, BedTools V2.25.0 softwares. RNA-seq data were analysed using STAR software. FACS data analyses were performed with the Flowjo v10 software.

For manuscripts utilizing custom algorithms or software that are central to the research but not yet described in published literature, software must be made available to editors/reviewers upon request. We strongly encourage code deposition in a community repository (e.g. GitHub). See the Nature Research guidelines for submitting code \& software for further information. 
Policy information about availability of data

All manuscripts must include a data availability statement. This statement should provide the following information, where applicable:

- Accession codes, unique identifiers, or web links for publicly available datasets

- A list of figures that have associated raw data

- A description of any restrictions on data availability

Data availability

Deep sequencing (Fig. 2 and 4) and ChIP-seq data (Fig. 2) that support the findings of this study have been deposited in GEO under accession code GSE102831..

Previously published sequencing data that were re-analysed here are available under accession code E-MTAB-2958, E-MTAB-2959 (ref. 31 ), GSE81285 (ref. 30 ) and GSE31381 (ref. 52).

All other data supporting the findings of this study are available from the corresponding author on reasonable request.

\section{Field-specific reporting}

Please select the best fit for your research. If you are not sure, read the appropriate sections before making your selection.

$\bigotimes$ Life sciences $\quad \square$ Behavioural \& social sciences $\square$ Ecological, evolutionary \& environmental sciences

For a reference copy of the document with all sections, see nature.com/authors/policies/ReportingSummary-flat.pdf

\section{Life sciences study design}

All studies must disclose on these points even when the disclosure is negative.

Sample size

Sample sizes were determined without statistical measures, but based on prior experience with the specific experiments and widely used sizes in relevant publications within this field of research in order to ensure that it will be appropriate for statistical analysis. See Figures legends for each experiment.

Data exclusions No data exclusion was used in the study.

Replication The vast majority of the presented results come from three independent biological replicates. RNA-seq and ChIP-seq data are coming from 3 independent biological replicates. RRBS data are coming from 2 independent biological replicates.

Randomization No randomization was used in the study.

Blinding Experiments execution, data collection and result analysis were usually carried out by the same researcher, therefore no blinding was used.

\section{Reporting for specific materials, systems and methods}

Materials \& experimental systems

\begin{tabular}{l|l}
\hline n/a Involved in the study \\
$\square$ Unique biological materials \\
$\square$ Antibodies
\end{tabular}

\begin{tabular}{l|l}
\multicolumn{2}{l}{ Methods } \\
\hline n/a Involved in the study \\
\hline
\end{tabular}

\section{Antibodies}

Antibodies used

Detailed information on antibody vendors, catalog and dilutions used can be found in Supplementary Table 1 or below: oct4, Santa Cruz, sc5279, 1/1000; Nanog, Cosmobio, RCA B000 2P-F, 1/1000; Sox2, Abcam, ab9759, 1/1000; Esrrb, R\&D Systems, PP-H6705-00, 1/2000; Actin, Sigma, A3854, 1/10000; Neo1, cell signalling, 39447, 1/1000; Unc5B, cell signalling, 13851, 1/1000 Netrin-1, R\&D Systems, AF-6419, 1/1000; p-ERK1/2, Cell Signaling, T202 Y204-9101, 1/1000; total ERK1/2, Sigma, M5670, 1/1000; p-STAT3, Tyr705, Cell signalling, D3A7, 1/1000; STAT3, Cell Signaling, 79D7, 1/1000; active- $\beta$-Catenin, Millipore, 05-665, 1/1000; TCF7L1, kind gift from B. Merrill lab, 1/1000; H3K27Me3, Diagenode \#C15410195-10, 1/1000; H3K4Me3, Active Motif \#61379, 1/1000; gsk3, cell signalling, 9315S, 1/1000; p-gsk3, 9331S cell signalling, 1/1000; mek1/2, cell signalling, 9122S, 
1/1000; p-mek1/2, cell signalling, 9121S, 1/1000; FAK, cell signalling, 3285S, 1/1000; p-FAK, cell signalling, 3283S, 1/1000; pp2AC millipore, 05-421, clone 1D6, 1/1000; total- $\beta$-Catenin, Cell signalling, 9562S, 1/1000.

Validation

The information of all antibodies we used can be found in their official website except for TCF7L1 that was validated in Yi et al., Nat cell biol 2011, PMID: 21685894.

\section{Eukaryotic cell lines}

Policy information about cell lines

Cell line source(s)

All the cell lines sources can be found in the methods. Cgr8 ES cells have been kindly provided by B. Pain Lab, Bron, France. E14 ES cells were provided by M. Elena Torres Padilla lab, Munich, Germany. Human iPS cell lines were derived from humand dermal fibroblasts (Merck 106-05A).

Authentication

The differentiation potential of the human iPS cell lines was authenticated by performing in vitro and in vivo differentiation assays. netrin-1 conditional KO ES cells were tested in vitro and in vivo for their ability to differentiate. The other cell lines were not authenticated.

Mycoplasma contamination

All cells are tested negative for mycoplasma contamination using MycoAlert Mycoplasma detection kit from Lonza.

Commonly misidentified lines

(See ICLAC register)

None of the cell lines used in this study is listed in the database of commonly

misidentified cell lines maintained by ICLAC.

\section{Animals and other organisms}

Policy information about studies involving animals; ARRIVE guidelines recommended for reporting animal research

Laboratory animals

Laboratory animals used in the study are described in the methods. Teratoma assays were performed with 7-week-old severe combined immunodeficient (SCID) female mice (CB17/SCID, Charles River). Netrin-1Bgeo reporter and netrin-1 conditional knockout mESCs were derived from C57/bl6 mixed background pregnant females. Blastocyst injections were done using BALB/ cANRj embryos.

Wild animals

The study did not involve wild animals.

Field-collected samples

The study did not involve samples collected from the field.

ChIP-seq

Data deposition

\Confirm that both raw and final processed data have been deposited in a public database such as GEO.

\Confirm that you have deposited or provided access to graph files (e.g. BED files) for the called peaks.

Data access links

May remain private before publication

Files in database submission

Genome browser session (e.g. UCSC)

Methodology

Replicates

Sequencing depth

Antibodies
All NGS data have been deposited in GEO accession number GSE102831 subserie GSE 105062.

Raw and processed data files:

GSM4103899 WT_H3K27me3 ChIP-seq rep1

GSM4103900 WT_H3K27me3 ChIP-seq rep2

GSM4103901 WT_H3K27me3 ChIP-seq rep3

GSM4103902 Netrin H3K27me3 ChIP-seq rep1

GSM4103903 Netrin_H3K27me3 ChIP-seq rep2

GSM4103904 Netrin H3K27me3 ChIP-seq rep3

GSM4103905 WT_H3K4me3 ChIP-seq rep1

GSM4103906 WT H3K4me3 ChIP-seq rep2

GSM4103907 WT_H3K4me3 ChIP-seq rep3

GSM4103908 Netrin_H3K4me3 ChIP-seq rep1

GSM4103909 Netrin_H3K4me3 ChIP-seq rep2

GSM4103910 Netrin_H3K4me3 ChIP-seq rep3

No

three replicates were analysed bor $\mathrm{H} 3 \mathrm{~K} 4 \mathrm{Me} 3$ and $\mathrm{H} 3 \mathrm{~K} 27 \mathrm{Me} 3$ in ctrl and netrin-1 WT expressing mESCs.

We sequenced an averaged of 38.6 million reads per sample.

H3K27Me3, Diagenode \#C15410195-10; H3K4Me3, Active Motif \#61379. 


\section{Flow Cytometry}

Plots

Confirm that:

$\bigotimes$ The axis labels state the marker and fluorochrome used (e.g. CD4-FITC).

$\bigotimes$ The axis scales are clearly visible. Include numbers along axes only for bottom left plot of group (a 'group' is an analysis of identical markers).

$\bigotimes$ All plots are contour plots with outliers or pseudocolor plots.

$\bigotimes$ A numerical value for number of cells or percentage (with statistics) is provided.

Methodology

Sample preparation

Instrument

Software

Cell population abundance

Gating strategy preparation Mouse ES cells were washed with PBS, trypsinized and resuspended in PBS with 5\% FBS for FACS.

Flow cytometry analysis was performed using the BD FACSCanto II and sorting was performed on the BD FACSAria II sorter.

BD FACSDiva (v8.0) was used for data collection and FlowJo (v10) was used for data analysis.

at least 10,000 events were quantified.

Stringent gatings were always used, leaving a significant gap in between negative/postive population. Cells sorted by FACS were reanalyzed with BD FACSCanto II to confirm the purity. Gating can be found in Extended Data Fig. 2e.

\Tick this box to confirm that a figure exemplifying the gating strategy is provided in the Supplementary Information. 